\title{
High-resolution smoothed particle hydrodynamics simulations of the merger of binary white dwarfs
}

\author{
P. Lorén-Aguilar ${ }^{1,2}$, J. Isern ${ }^{3,2}$, and E. García-Berro ${ }^{1,2}$ \\ 1 Departament de Física Aplicada, Escola Politécnica Superior de Castelldefels, Universitat Politècnica de Catalunya, Avda. del Canal \\ Olímpic 15, 08860 Castelldefels, Spain \\ e-mail: garcia@fa.upc.es \\ 2 Institute for Space Studies of Catalonia, c/Gran Capità 2-4, Edif. Nexus 104, 08034 Barcelona, Spain \\ 3 Institut de Ciències de l'Espai, CSIC, Campus UAB, Facultat de Ciències, Torre C-5, 08193 Bellaterra, Spain
}

Received 30 September 2008 / Accepted 15 March 2009

\section{ABSTRACT}

\begin{abstract}
Context. The coalescence of two white dwarfs is the final outcome of a sizeable fraction of binary stellar systems. Moreover, this process has been proposed to explain several interesting astrophysical phenomena.

Aims. We present the results of a set of high-resolution simulations of the merging process of two white dwarfs.

Methods. We use an up-to-date smoothed particle hydrodynamics code that incorporates very detailed input physics and an improved treatment of the artificial viscosity. Our simulations have been done using a large number of particles $\left(\sim 4 \times 10^{5}\right)$ and covering the full range of masses and chemical compositions of the coalescing white dwarfs. We also compare the time evolution of the system during the first phases of the coalescence with what is obtained using a simplified treatment of mass transfer; we discuss in detail the characteristics of the final configuration; we assess the possible observational signatures of the merger, such as the associated gravitational waveforms and the fallback X-ray flares; and we study the long-term evolution of the coalescence.

Results. The mass transfer rates obtained during the first phases of the merger episode agree with the theoretical expectations. In all the cases studied, the merged configuration is a central compact object surrounded by a self-gravitating Keplerian disk, except in the case where two equal-mass white dwarfs coalesce.

Conclusions. We find that the overall evolution the system and the main characteristics of the of the final object agree with other previous studies in which lower resolutions were used. We also find that the fallback X-ray luminosities are close to $10^{47} \mathrm{erg} / \mathrm{s}$. The gravitational waveforms are characterized by the sudden disappearance of the signal in a few orbital periods.
\end{abstract}

Key words. stars: white dwarfs - stars: interiors - stars: binaries: close - hydrodynamics - accretion, accretion disks

\section{Introduction}

The coalescence of two close white dwarfs is thought to be one of the most common endpoints in the evolution of binary systems. Consequently, the coalescence process is an interesting issue with many potential applications. Although the astrophysical scenarios in which the coalescence of two white dwarfs in a close binary system can occur and their relative frequencies have been often studied - see, for instance, Yungelson et al. (1994), Nelemans et al. (2001a,b), and the recent review of Postnov \& Yungelson (2006) - the merging process has received little attention until recently. The pioneering works of Mochkovitch \& Livio $(1989,1990)$ who used an approximate method - the so-called self-consistent-field method (Clement 1974) - and the full smoothed particle hydrodynamic ( $\mathrm{SPH}$ ) simulations of Benz et al. (1989a), Benz et al. (1989a), Benz et al. (1989a), Benz et al. (1990), Rasio \& Shapiro (1995) and Segretain et al. (1997) were the only exceptions.

Most of these early works had several drawbacks. For instance, some of them did not include a detailed nuclear network, or else the network was very simplistic; others used a very small number of SPH particles $\left(\sim 10^{3}\right)$ and, finally, others did not discuss the properties of the merger configuration. Additionally, all these early works studied a reduced set of masses and chemical compositions and used the classical expression for the artificial viscosity (Monaghan \& Gingold 1983). This is an important issue since it is well known that SPH induces a strong shear viscosity, which is more pronounced when the classical expression for the artificial viscosity is used. Likewise, energy dissipation by artificial viscosity can lead to overheating so the peak temperatures achieved during the merger and the associated nucleosynthesis depend on the choice of the artificial viscosity. However, the situation changed recently, when Guerrero et al. (2004) opened the way to more realistic simulations. In these calculations the standard prescription of Monaghan \& Gingold (1983) for the artificial viscosity was used, but the switch originally suggested by Balsara (1995) was employed to partially suppress the excess of dissipation. More recently, the simulations of Yoon et al. (2007) were carried out using a modern prescription for the artificial viscosity with time-dependent parameters (Morris \& Monaghan 1997), which guarantees that viscosity is essentially absent in those parts of the fluid in which it is not necessary, but there are other prescriptions that are also suitable. In particular, the prescription of Monaghan (1997), which is based in Riemann-solvers also yields excellent results, and does not result in overheating. This is the prescription we use in the present work.

It is also interesting to realize that the number of particles used in these kind of simulations has increased considerably in the last few years, according to the available computing power. For instance, Segretain et al. (1997) used $\sim 6 \times 10^{4} \mathrm{SPH}$ 
particles to simulate the coalescence of a $0.9+0.6 M_{\odot}$ system. Later, Guerrero et al. (2004) studied a considerable range of masses and chemical compositions of the merging white dwarfs employing a sizeable number of particles $\left(\sim 4 \times 10^{4}\right)$. This range of masses and chemical compositions included 6 runs in which several inital configurations were studied, involving helium, carbon-oxygen, and oxygen-neon white dwarfs. Lorén-Aguilar et al. (2005) simulated the coalescence of a system of two equalmass carbon-oxygen white dwarfs of $0.6 M_{\odot}$. However, in this work only one high-resolution simulation was done. More recently, Yoon et al. (2007) studied in detail the coalescence of a binary system composed of two white dwarfs of masses 0.6 and $0.9 M_{\odot}$ using $2 \times 10^{5} \mathrm{SPH}$ particles. However, only one simulation was presented in this work. It is thus clear that a thorough parametric study in which several white dwarf masses and chemical compositions are explored using a large number of SPH particles and a more elaborate treatment of the artificial viscosity remains to be done.

Possible applications of these kind of simulations include the double-degenerate scenario to account for Type Ia supernova outbursts (Webbink 1984; Iben \& Tutukov 1984) and the formation of magnetars (King et al. 2001). Also, three hot and massive white dwarfs members of the Galactic halo could be the result of the coalescence of a double white-dwarf binary system (Schmidt et al. 1992; Segretain et al. 1997). Additionally, hydrogendeficient carbon and R Corona Borealis stars (Izzard et al. 2007; Clayton et al. 2007) and extreme helium stars (Pandey et al. 2005) are thought to be the consequence of the merging of two white dwarfs. Finally, the large metal abundances found around some hydrogen-rich white dwarfs with dusty disks around them can be explained by the merger of a $\mathrm{CO}$ and a He white dwarf (García-Berro et al. 2007). Last but not least, the phase previous to the coalescence of a double white-dwarf close binary system has been shown to be a powerful source of gravitational waves that would be eventually detectable by LISA (Lorén-Aguilar et al. 2005).

Depending on the mass ratio of both stars and on the initial conditions of the binary system, the fate of double white dwarf binary systems is a merging process because angular momentum losses through gravitational wave radiation. Stars orbit each other at decreasing orbital separations until the less massive one overfills its Roche lobe and mass transfer begins. According to the initial conditions, mass transfer proceeds either in a stable or a dynamically unstable regime. The stability of mass transfer is an important issue. If the mass transfer process is stable, mass will flow at relatively low accretion rates, and the whole merging process could last for several million years. In contrast, if mass transfer proceds in an unstable way, the whole merging process finishes in a few minutes. The difference between the two cases relies on the ability of the binary system to return enough angular momentum back to the orbit. In fact, there are two competing processes. On the one hand, the donor star is supported by the pressure of degenerate electrons, so it will expand as it loses mass, thus enhancing the mass-transfer rate. On the other, if orbital angular momentum is conserved, the orbit will expand as the donor star loses mass, thus reducing the masstransfer rate. The precise trade-off between both physical processes determines the stability of mass transfer. Guerrero et al. (2004) find that all the systems merged in a few hundred seconds, corresponding to mass transfer rates of $\sim 10^{-2} M_{\odot} \mathrm{s}^{-1}$. Since the Eddington rate is about $10^{-5} M_{\odot} \mathrm{yr}^{-1}$, the most massive white dwarf cannot incorporate the material of the disrupted secondary on such a short timescale and, thus, the secondary forms a hot atmosphere and a heavy Keplerian disk around the primary. This has been challenged by the simulations of Motl et al. (2002) and D'Souza et al. (2006). These authors used a grid-based, threedimensional finite-difference Eulerian hydrodynamical code and found that mass transfer is stable when the stars are co-rotating. Nevertheless, it should be noted that these simulations were done using simplified physical inputs; for instance, they used a polytropic equation of state. More importantly, grid-based methods are known to poorly conserve angular momentum. In either case, it is clear that large spatial resolutions are required to assess the stability of mass transfer given the degenerate nature of the donor star, since once mass transfer begins, the radius of the secondary increases very rapidly, thereby increasing the mass-loss rate.

In the present paper we study the coalescence of binary white dwarfs employing an enhanced spatial resolution $\left(4 \times 10^{5} \mathrm{SPH}\right.$ particles) and a formulation of the artificial viscosity which very much reduces the excess of shear. The number of particles used in our simulations is one order of magnitude greater than those used in our previous simulations (Guerrero et al. 2004) and within a factor of 2 of those used in modern simulations (Yoon et al. 2007; Lorén-Aguilar et al. 2005). This allows us to resolve smaller scale lengths - by a factor $\left(10^{5} / 10^{4}\right)^{1 / 3} \approx 2-$ than in Guerrero et al. (2004). Moreover, this is done for a broad range of initial masses and chemical compositions of the coalescing white dwarfs, in contrast to most modern simulations, in which only a single coalescence was studied in detail (Yoon et al. 2007). In particular we study the following cases: $0.3+0.5 M_{\odot}$, $0.4+0.8 M_{\odot}, 0.6+0.6 M_{\odot}, 0.6+0.8 M_{\odot}$ and $0.6+1.2 M_{\odot}$. Although we have computed several mergers, we only discuss in detail the results of the merger of a $0.6+0.8 M_{\odot}$ binary system. The main results of the rest of the simulations are only given in tabular form, but can be provided upon request. Accordingly, we devote most of the paper to compare the results of this simulation with those previously available, and we postpone the discussion of the effects of the initial conditions to a forthcoming publication. The paper is organized as follows. A brief description of our SPH code is given in Sect. 2. In Sect. 3 we describe our results and in Sect. 4 we compare them with those of other authors. In Sect. 5 we discuss our simulations. Specifically, we pay special attention to the stability of the mass transfer episode. This is done in Sect. 5.1, whereas in Sects. 5.2 and 5.3 the possible observational signatures arising from the merging process are studied. In particular, we consider the gravitational wave pattern of the several mergers studied here (Sect. 5.2) and the X-ray emission that might be expected from the early phases of the disk evolution (Sect. 5.3), while in Sect. 5.4 the long-term evolution of the merger is discussed. Finally, in Sect. 6 we summarize our major findings, elaborate on the possible implications of our work, and draw our conclusions.

\section{Input physics and method of calculation}

We follow the hydrodynamic evolution of the binary system using a Lagrangian particle numerical code, the so-called smoothed particle hydrodynamics. This method was first proposed by Lucy (1977) and, independently, by Gingold \& Monaghan (1977). That the method is totally Lagrangian and does not require a grid makes it specially suitable for studying an intrinsically three-dimensional problem like the coalescence of two white dwarfs. We will not describe in detail the most basic equations of our numerical code, since this is a wellknown technique. Instead, the reader is referred to Benz (1990) where the basic numerical scheme for solving the hydrodynamic equations can be found, whereas a general introduction to the 
SPH method can be found in the excellent review of Monaghan (2005). However, and for the sake of completeness, we briefly describe the most relevant equations of our numerical code.

We use the standard polynomic kernel of Monaghan \& Lattanzio (1985). The gravitational forces are evaluated using an octree (Barnes \& Hut 1986). Our SPH code uses a prescription for the artificial viscosity based in Riemann-solvers (Monaghan 1997). Additionally, to suppress artificial viscosity forces in pure shear flows, we also use the viscosity switch of Balsara (1995). In this way the dissipative terms are essentially absent in most parts of the fluid and are only used where they are really necessary to resolve a shock, if present. Within this approach, the SPH equations for the momentum and energy conservation read, respectively, as

$$
\begin{aligned}
\frac{\mathrm{d} \boldsymbol{v}_{i}}{\mathrm{~d} t} & =-\sum_{j} m_{j}\left(\frac{P_{i}}{\rho_{i}^{2}}+\frac{P_{j}}{\rho_{j}^{2}}-\alpha \frac{v_{i j}^{\mathrm{sig}}}{\bar{\rho}_{i j}} \boldsymbol{v}_{i j} \cdot \hat{e}_{i j}\right) \boldsymbol{r}_{i j} F_{i j} \\
\frac{\mathrm{d} u_{i}}{\mathrm{~d} t} & =\frac{P_{i}}{\rho_{i}^{2}} \sum_{j} m_{j} \boldsymbol{v}_{i j} \cdot \boldsymbol{r}_{i j} \bar{F}_{i j}-\frac{1}{2} \sum_{j} m_{j} \alpha \frac{v_{i j}^{\mathrm{sig}}}{\bar{\rho}_{i j}}\left(\boldsymbol{v}_{i j} \cdot \hat{e}_{i j}\right)^{2}\left|\boldsymbol{r}_{i j}\right| \bar{F}_{i j}
\end{aligned}
$$

where $\bar{\rho}_{i j}=\left(\rho_{i}+\rho_{j}\right) / 2$ and $\overline{F_{i j}} \equiv\left(F_{i}+F_{j}\right) / 2$, and $F$ is a positive definite function that only depends on $|\boldsymbol{r}|$ and on the smoothing kernel $h$, used to express gradient of the kernel $\nabla W_{i j}=\bar{F}_{i j} \boldsymbol{r}_{i j}$. The signal velocity is taken as $v_{i j}^{\text {sig }}=c_{i}+c_{j}-4 v_{i j} \cdot \hat{e}_{i j}$ and the rest of the symbols have their usual meaning. We find that $\alpha=0.5$ yields good results.

We have found that it is sometimes advisable to use a different formulation of the equation of energy conservation. Accordingly, for each time step we compute the variation of the internal energy using Eq. (2) and simultaneously calculate the variation of temperature using

$\frac{\mathrm{d} T_{i}}{\mathrm{~d} t}=-\sum_{j=1}^{N} \frac{m_{j}}{\left(C_{v}\right)_{j}} \frac{T_{j}}{\rho_{i} \rho_{j}}\left[\left(\frac{\partial P}{\partial T}\right)_{\rho}\right]_{j} \boldsymbol{v}_{i j} \cdot \nabla_{i} W\left(\left|\boldsymbol{r}_{i j}\right|, h\right)+q_{\mathrm{visc}}$

where $q_{\text {visc }}$ includes the contribution of viscous dissipation, which is computed analogously to that of Eq. (2). For regions in which the temperatures are lower than $6 \times 10^{8} \mathrm{~K}$ or the densities are lower than $6 \times 10^{3} \mathrm{~g} / \mathrm{cm}^{3} \mathrm{Eq}$. (2) is adopted, whereas Eq. (3) is used in the rest of the fluid. Using this prescription we find that energy is best conserved.

The equation of state adopted for the white dwarf is the sum of three components. The ions are treated as an ideal gas but take the Coulomb corrections into account (Segretain et al. 1994). We have also incorporated the pressure of photons, which turns out to be important when the temperature is high and the density is small, just when nuclear reactions become relevant. Finally the most important contribution is the pressure of degenerate electrons, which is treated by integrating the Fermi-Dirac integrals. The nuclear network adopted here (Benz et al. 1989a) incorporates 14 nuclei: $\mathrm{He}, \mathrm{C}, \mathrm{O}, \mathrm{Ne}, \mathrm{Mg}, \mathrm{Si}, \mathrm{S}, \mathrm{Ar}, \mathrm{Ca}, \mathrm{Ti}, \mathrm{Cr}, \mathrm{Fe}, \mathrm{Ni}$, and $\mathrm{Zn}$. The reactions considered are captures of $\alpha$ particles, and the associated back reactions, the fussion of two $\mathrm{C}$ nuclei, and the reaction between $\mathrm{C}$ and $\mathrm{O}$ nuclei. All the rates are taken from Rauscher \& Thielemann (2000). The screening factors adopted in this work are those of Itoh et al. (1979). The nuclear energy release is computed independently of the dynamical evolution with much shorter time steps, assuming that the dynamical variables do not change much during these time steps. Finally, neutrino losses have also been included according to the formulation of
Itoh et al. (1996) for the pair, photo, plasma, and bremsstrahlung neutrino processes.

For the integration method, we use a predictor-corrector numerical scheme with variable time steps (Serna 1996), which turns out to be quite accurate. Each particle is followed by individual time steps. With this procedure the energy and angular momentum of the system are conserved with good accuracy. To avoid numerical artifacts, we only use equal-mass SPH particles, as in Yoon et al. (2007). This was not the case for the simulations of Guerrero et al. (2004) in which the masses of the SPH particles were different for each one of the coalescing white dwarfs. To achieve an equilibrium initial configuration, we relaxed each individual model star separately, so the two coalescing white dwarfs are spherically symmetric at the beginning of our simulations, as was the case in all previous simulations of this kind. In all the cases the two white dwarfs are initially in a circular orbit at a greater distance than the corresponding Roche lobe radius of the less massive component. The systems are not synchronized because, at least in the stage previous to the coalescence itself, the time scale for loss of angular momentum due to the emission of gravitational radiation is so small that it remains quite unlikely that there exists any dissipation mechanism able to ensure synchronization (Segretain et al. 1997). This is the same initial configuration adopted by Yoon et al. (2007), Guerrero et al. (2004), and Segretain et al. (1997). However, as already stated, we will study synchronized systems in a forthcoming publication. To this system we add a very small artificial acceleration term that decreases the separation of both components. Once the secondary fills its Roche lobe, this acceleration term is suppressed. This procedure is quite similar to the one adopted in all previous works (Guerrero et al. 2004; Yoon et al. 2007; Segretain et al. 1997). We adopt this instant as our time origin.

The chemical compositions of the coalescing white dwarfs depend on the mass of each star. White dwarfs with masses lower than $0.45 M_{\odot}$ have pure He cores. For white dwarfs with masses within this value and $1.1 M_{\odot}$ we adopt the corresponding chemical composition, namely, carbon and oxygen, with mass fractions $X_{\mathrm{C}}=0.4$ and $X_{\mathrm{O}}=0.6$ uniformingly distributed throughout the core. Finally, white dwarfs more massive than $1.1 M_{\odot}$ have ONe cores of the appropriate composition (Ritossa et al. 1996). This is essential in studying the resulting chemical composition of the merger, as shown in Sect. 3, and it is a clear improvement over recent high-resolution simulations (Yoon et al. 2007) in which only a single system of two carbon-oxygen white dwarfs was studied.

\section{Results}

Figure 1 shows the temporal evolution of the logarithm of the density for the coalescence of the $0.6+0.8 M_{\odot}$ white dwarf binary system. In the left panels the positions of the SPH particles have been projected onto the equatorial plane and in the right panels onto the polar plane. Time (in seconds) is shown in the upper right corner of each panel. As can be seen in the uppermost left panels, the initial configurations of both white dwarfs are symmetric. Soon after, the less massive white dwarf fills its Roche lobe and mass tranfer begins, as can be seen in the top central panel of this figure. The top right panel of Fig. 1 shows that, after some time, the matter flowing out of the secondary hits the surface of the primary white dwarf and spreads on top of it. We note as well that since the radius of white dwarfs scales as $\sim M^{-1 / 3}$ and since the secondary loses mass its radius increases and, hence, the mass-loss rate of the secondary increases, thus 
$\log \rho$

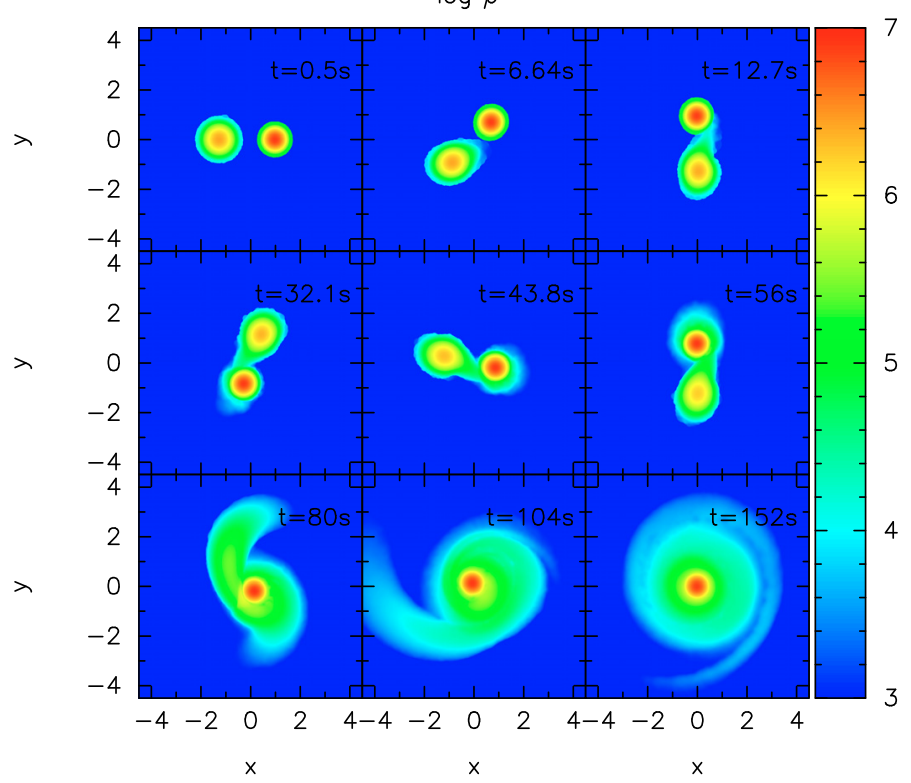

$\log \rho$

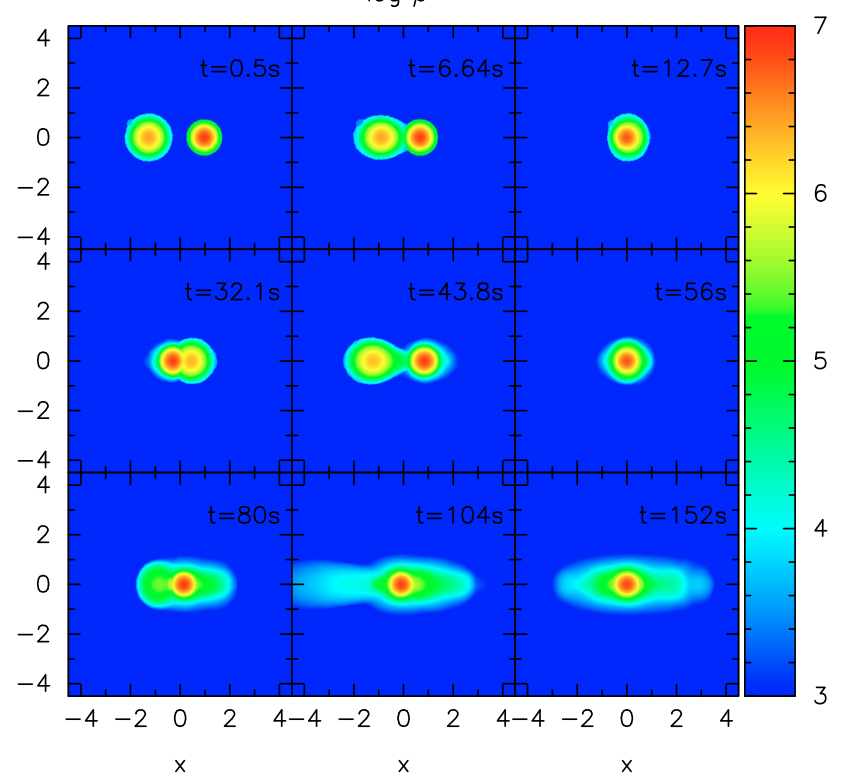

Fig. 1. Temporal evolution of the density for the coalescence of the $0.6+0.8 M_{\odot}$ double white-dwarf binary system. The positions of the particles have been projected onto the $x y$ plane (left panels) and on the $x z$ plane (right panels). The units of positions and densities are, respectively, $10^{9} \mathrm{~cm}$ and $10^{9} \mathrm{~g} / \mathrm{cm}^{3}$. Times are shown in the right upper corner of each panel. These figures have been done using the visualization tool SPLASH (Price 2007). (Color figure only available in the electronic version of the article).

leading to a positive feedback of the process. As a consequence of this positive feedback an accretion arm is formed that extends from the remnant of the secondary white dwarf (central panels in Fig. 1) to the surface of the primary white dwarf. This accretion arm becomes entangled as a consequence of the orbital motion of the coalescing white dwarfs and adopts a spiral shape (bottom left panel). Ultimately, the secondary is totally disrupted and a heavy disk is formed around the primary (bottom central panel of Fig. 1). The bottom right panel of Fig. 1 shows that at time $t=152 \mathrm{~s}$ the disk is still not well formed and the remnant of a spiral arm still persists. We followed the evolution of this merger for some more time and we found that the final configuration has cylindrical symmetry, that most of the orbits of the SPH particles belonging to the secondary have been circularized, and that the spiral pattern has totally disappeared. At the end of the simulations the radial extension of the disk is $\sim 0.2 R_{\odot}$, whereas its height is $\sim 5.0 \times 10^{-3} R_{\odot}$.

The temporal evolution of the temperature for the merger of a $0.6+0.8 M_{\odot}$ binary system is shown in Fig. 2. As can be seen in this figure, the material of the secondary is first heated by tidal torques. As the secondary begins the disruption process this material is transferred to the surface of the primary and, consequently, compressed, and its temperature increases. The peak temperatures $\left(T_{\text {peak }}\right)$ achieved during the coalescence are displayed in Table 1 for each one of the runs presented in this paper. For the $0.6+0.8 M_{\odot}$ simulation, the peak temperature is $T_{\text {peak }} \sim 1.6 \times 10^{9} \mathrm{~K}$, clearly higher than the carbon ignition temperature $T_{\text {ign }} \sim 10^{9} \mathrm{~K}$, and occurs during the first and most violent part of the merger. However, a strong thermonuclear flash does not develop because, although the temperature in the region where the material of the secondary first hits the primary increases very rapidly, degeneracy is rapidly lifted, leading to an expansion of the material, which, in turn, quenches the thermonuclear flash. This agrees with the results of Guerrero et al. (2004) and Yoon et al. (2007). Thus, since these high temperatures are only attained during a very short time interval, thermonuclear processing is very mild for this simulation. It is also interesting to compare the equatorial and polar distribution of temperatures shown in the central panels of Fig. 2. This comparison reveals that the heated material is rapidly redistributed on the surface of the primary, and as a consequence, a hot corona forms around the primary. The spiral structure previously described can be more easily appreciated in the bottom right panels of Fig. 2. In fact, this spiral structure persists for some more time.

In all the cases studied here, a self-gravitating structure forms after a few orbital periods, in agreement with our previous findings (Guerrero et al. 2004) and with those of Yoon et al. (2007). The time necessary for its formation depends on the system being studied and ranges from $\sim 120 \mathrm{~s}$ to $\sim 520 \mathrm{~s}$. This selfgravitating structure consists in all the cases but that in which two equal-mass white dwarfs are involved in a compact central object, surrounded by a heavy keplerian disk of variable extension. The case in which two $0.6 M_{\odot}$ white dwarfs are involved the configuration is rather different. There the symmetry of the systems avoids the formation of a clear disk structure, instead giving rise to a rotating elipsoid around the central compact object, surrounded by a considerably smaller disk. In Table 1 we summarize the most relevant parameters of all the mergers studied here. Columns two, three, four, and five list, respectively, the mass of the central white dwarf obtained after the disruption of the secondary, the mass of the Keplerian disk, the accreted and the ejected mass. All the masses are expressed in solar units. In column six we show the peak temperatures achieved during the coalescence. In column seven we display the temperature of the hot corona around the central object by the end of our simulations, whereas in column eight the radius of the disk is shown. In column nine we list the disk half thickness. Column ten displays the duration of the coalescence process and columns eleven, twelve, and thirteen display the energetics of the process. In particular we show the thermonuclear energy released during the coalescence process $\left(E_{\text {nuc }}\right)$, the neutrino energy $\left(E_{\gamma}\right)$, and 
$\mathrm{T}$

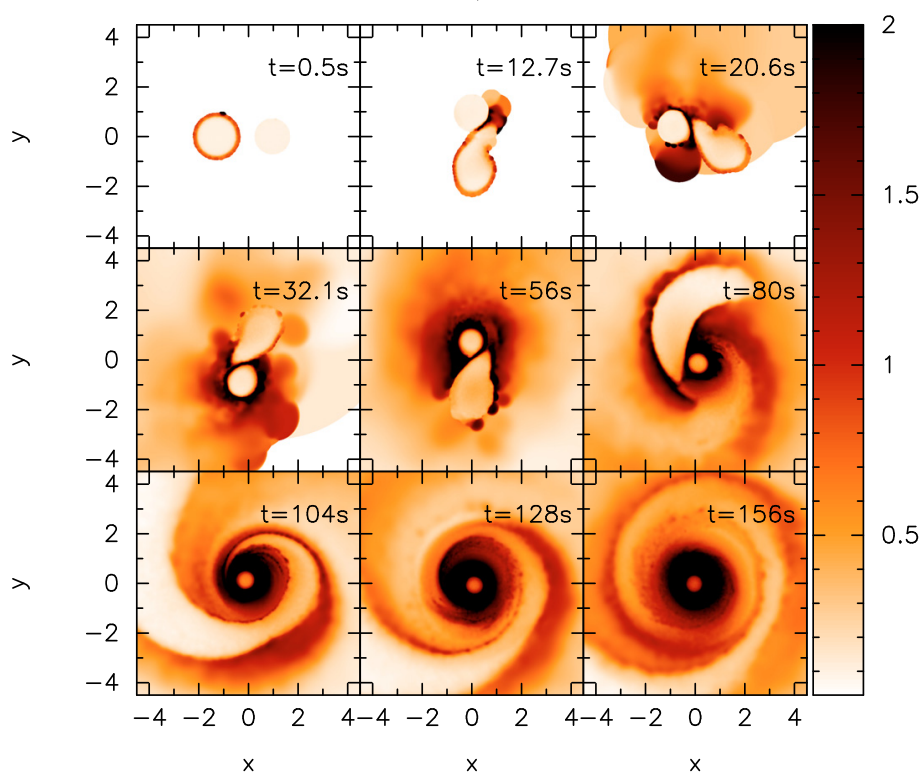

T

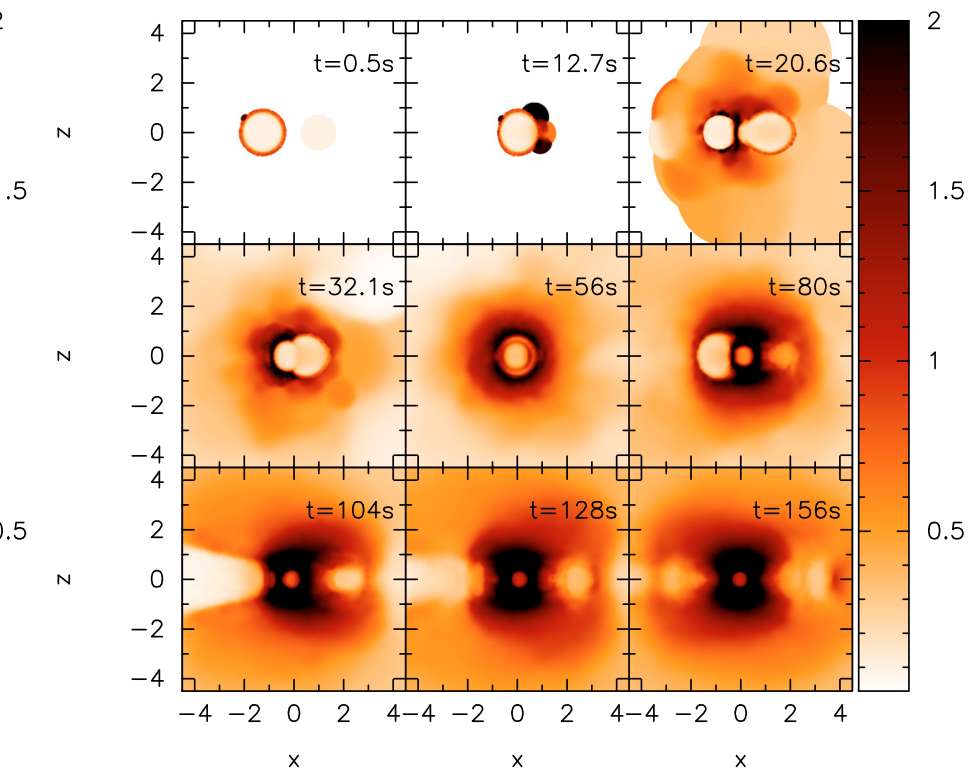

Fig. 2. Temporal evolution of the temperature (in units of $10^{9} \mathrm{~K}$ ) for the coalescence of the same binary system shown in Fig. 1. The positions of the particles have been projected onto the $x y$ plane (left panels) and on the $x z$ plane (right panels). These figures use the visualization tool SPLASH (Price 2007). (Color figure only available in the electronic version of the article).

Table 1. Summary of hydrodynamical results.

\begin{tabular}{cccccccccccccc}
\hline \hline Run & $M_{\mathrm{WD}}$ & $M_{\text {disk }}$ & $M_{\mathrm{acc}}$ & $M_{\mathrm{ej}}$ & $T_{\text {peak }}$ & $T_{\max }$ & $R_{\text {disk }}$ & $H$ & $\Delta t$ & $E_{\text {nuc }}$ & $E_{v}$ & $E_{\mathrm{GW}}$ & $\omega$ \\
\hline $0.3+0.5$ & 0.62 & 0.18 & 0.12 & $10^{-3}$ & $6.0 \times 10^{8}$ & $6.0 \times 10^{8}$ & 0.2 & $6.1 \times 10^{-3}$ & 300 & $1 \times 10^{42}$ & $4 \times 10^{21}$ & $9 \times 10^{38}$ & 0.14 \\
$0.4+0.8$ & 0.92 & 0.28 & 0.12 & $10^{-3}$ & $6.5 \times 10^{8}$ & $6.0 \times 10^{8}$ & 0.2 & $6.0 \times 10^{-3}$ & 166 & $1 \times 10^{44}$ & $5 \times 10^{24}$ & $4 \times 10^{39}$ & 0.23 \\
$0.6+0.6$ & 1.10 & 0.10 & 0.50 & $10^{-3}$ & $6.3 \times 10^{8}$ & $6.2 \times 10^{8}$ & 0.07 & $5.5 \times 10^{-3}$ & 514 & 0 & $3 \times 10^{24}$ & $1 \times 10^{41}$ & 0.27 \\
$0.6+0.8$ & 1.10 & 0.30 & 0.30 & $10^{-3}$ & $1.6 \times 10^{9}$ & $8.7 \times 10^{8}$ & 0.2 & $5.0 \times 10^{-3}$ & 164 & $1 \times 10^{41}$ & $3 \times 10^{28}$ & $6 \times 10^{40}$ & 0.33 \\
$0.6+1.2$ & 1.50 & 0.30 & 0.30 & $10^{-3}$ & $1.0 \times 10^{10}$ & $1.0 \times 10^{9}$ & 0.2 & $4.4 \times 10^{-3}$ & 122 & $2 \times 10^{44}$ & $8 \times 10^{36}$ & $5 \times 10^{40}$ & 0.52 \\
\hline
\end{tabular}

Note: masses and radii are in solar units, times in seconds and energies in ergs. The maximum temperature achieved during each simulation, $T_{\text {peak }}$, and the temperature of the hot corona formed around the primary at the end of the simulations, $T_{\max }$, are discussed in the text. The rotational velocity is expressed in $\mathrm{s}^{-1}$.

the energy radiated in the form of gravitational waves $\left(E_{\mathrm{GW}}\right)$. Finally, in column fourteen we list the angular velocities of the central compact remnants.

As can be seen, for the first two simulations the accreted mass is approximately the same and the same occurs for the last two simulations. As already said, the simulation in which two equal-mass white dwarfs are involved is rather special and in this case we do not have a disk properly speaking, although a very flattened region with cylindrical symmetry forms around a central object of ellipsoidal shape. The mass of this region is $\sim 0.1 M_{\odot}$. In all five cases, the mass ejected from the system (those particles which acquire velocities higher than the escape velocity) is very low $\left(\sim 10^{-3} M_{\odot}\right)$, and thus the merging process can be considered as conservative. The maximum temperatures of the coronae increase as the total mass of the binary system increases. It should be noted that, for the case of the $0.6+0.6 M_{\odot}$ binary system, the maximum temperature occurs at the center of the merged configuration. We have found that these temperatures are somewhat lower than those obtained in our previous simulations (Guerrero et al. 2004). This is a direct consequence of the improved treatment of the artificial viscosity and of the enhanced spatial resolution. For instance, we have found that by using an enhanced resolution and an improved prescription for the viscosity, the peak temperature obtained in the simulation in which a 0.6 and $0.8 M_{\odot}$ are involved is $T_{\text {peak }} \simeq 1.64 \times 10^{9} \mathrm{~K}-$ see Table 1 . When a reduced number of SPH particles and the classical expression for the artificial viscosity are used, this temperature is $T_{\text {peak }} \simeq 1.72 \times 10^{9} \mathrm{~K}$, whereas the peak temperature turns out to be $T_{\text {peak }} \simeq 1.68 \times 10^{9} \mathrm{~K}$ when a reduced number of particles and an improved artificial viscosity are used. It is worth noting that the radial extension of the disks is roughly the same for all but one the simulations presented here, and it is considerably smaller for the case in which two equal-mass white dwarfs are involved. This is a natural behavior since the central object is rather massive in this last case. Finally, it is just as interesting to realize that all the disks are rather thin, being the typical half-thickness on the order of $\sim 10^{-3} R_{\odot}$, much smaller than the typical disk radial extension, $\sim 0.2 R_{\odot}$.

The chemical composition of the disk formed by the disrupted secondary can be found for all the simulations presented in this paper in Table 2. In this table we show, for each of the mergers computed here, the averaged chemical composition (mass fractions) of the heavily rotationally-supported disk - left section of Table 2 - and the hot corona - right section - described previously. For the mergers in which two carbon-oxygen white dwarfs are involved, the disk is mainly formed by carbon and oxygen and the nuclear processing is very small (see the peak temperatures shown in column ten of Table 1). This is not 
Table 2. Averaged chemical composition (mass fractions) of the heavy rotationally-supported disk and the hot corona obtained by the end of the coalescing process.

\begin{tabular}{lcccccccccc}
\hline \hline & \multicolumn{3}{c}{ Disk } & & \multicolumn{5}{c}{ Corona } \\
\hline Run & $0.3+0.5$ & $0.4+0.8$ & $0.6+0.6$ & $0.6+0.8$ & $0.6+1.2$ & $0.3+0.5$ & $0.4+0.8$ & $0.6+0.6$ & $0.6+0.8$ & $0.6+1.2$ \\
\hline $\mathrm{He}$ & 0.94 & 0.98 & 0 & 0 & $5 \times 10^{-5}$ & 0.72 & 0.68 & 0 & 0 & $5 \times 10^{-5}$ \\
$\mathrm{C}$ & $2.4 \times 10^{-2}$ & $7 \times 10^{-3}$ & 0.4 & 0.4 & 0.39 & 0.11 & 0.12 & 0.4 & 0.4 & 0.26 \\
$\mathrm{O}$ & $3.6 \times 10^{-2}$ & $9 \times 10^{-3}$ & 0.6 & 0.6 & 0.60 & 0.17 & 0.18 & 0.6 & 0.6 & 0.66 \\
$\mathrm{Ne}$ & $9 \times 10^{-13}$ & $6 \times 10^{-10}$ & 0 & 0 & $3 \times 10^{-3}$ & $3 \times 10^{-13}$ & $7 \times 10^{-7}$ & 0 & 0 & 0.07 \\
$\mathrm{Mg}$ & $4 \times 10^{-14}$ & $5 \times 10^{-11}$ & 0 & 0 & $7 \times 10^{-5}$ & $1 \times 10^{-12}$ & $1 \times 10^{-5}$ & 0 & 0 & $8 \times 10^{-5}$ \\
$\mathrm{Si}$ & $1 \times 10^{-17}$ & $3 \times 10^{-14}$ & 0 & 0 & $1 \times 10^{-5}$ & $1 \times 10^{-11}$ & $1 \times 10^{-4}$ & 0 & 0 & $5 \times 10^{-5}$ \\
$\mathrm{~S}$ & $2 \times 10^{-23}$ & $1 \times 10^{-19}$ & 0 & 0 & $3 \times 10^{-7}$ & $1 \times 10^{-10}$ & $2 \times 10^{-4}$ & 0 & 0 & $5 \times 10^{-5}$ \\
$\mathrm{Ar}$ & $<10^{-30}$ & $7 \times 10^{-27}$ & 0 & 0 & $1 \times 10^{-7}$ & $5 \times 10^{-9}$ & $9 \times 10^{-4}$ & 0 & 0 & $4 \times 10^{-5}$ \\
$\mathrm{Ca}$ & $<10^{-30}$ & $<10^{-30}$ & 0 & 0 & $8 \times 10^{-7}$ & $1 \times 10^{-8}$ & $5 \times 10^{-4}$ & 0 & 0 & $1 \times 10^{-4}$ \\
$\mathrm{Ti}$ & $<10^{-30}$ & $<10^{-30}$ & 0 & 0 & $7 \times 10^{-7}$ & $2 \times 10^{-4}$ & $1 \times 10^{-2}$ & 0 & 0 & $1 \times 10^{-4}$ \\
$\mathrm{Cr}$ & $<10^{-30}$ & $<10^{-30}$ & 0 & 0 & $8 \times 10^{-7}$ & $4 \times 10^{-4}$ & $2 \times 10^{-3}$ & 0 & 0 & $2 \times 10^{-4}$ \\
$\mathrm{Fe}$ & $<10^{-30}$ & $<10^{-30}$ & 0 & 0 & $5 \times 10^{-6}$ & $2 \times 10^{-5}$ & $1 \times 10^{-5}$ & 0 & 0 & $6 \times 10^{-4}$ \\
$\mathrm{Ni}$ & $<10^{-30}$ & $<10^{-30}$ & 0 & 0 & $6 \times 10^{-4}$ & $2 \times 10^{-7}$ & $4 \times 10^{-8}$ & 0 & 0 & $1 \times 10^{-2}$ \\
$\mathrm{Zn}$ & $<10^{-30}$ & $<10^{-30}$ & 0 & 0 & $6 \times 10^{-6}$ & $2 \times 10^{-9}$ & $6 \times 10^{-10}$ & 0 & 0 & $2 \times 10^{-5}$ \\
\hline
\end{tabular}

the case for the simulations in which a lighter He white dwarf is involved. Since in these cases the Coulomb barrier is considerably smaller, the shocked material is nuclearly processed and heavy isotopes form. This is more evident for the case in which a massive He white dwarf of $0.4 M_{\odot}$ is disrupted by a massive $\mathrm{CO}$ white dwarf of $0.8 M_{\odot}$ - third and eight columns in Table 2. In this case the abundances in the disk and the hot corona are rather large. Also, the abundances of heavy nuclei in the hot corona are much larger than those of the disk, indicating that most of the nuclear reactions occur when the accretion stream hits the surface of the primary.

Although the disk is primarily made of the He coming from the disrupted secondary, the abundances of $\mathrm{C}$ and $\mathrm{O}$ are sizeable; moreover, the disk is contaminated by heavy metals. This has important consequences because it is thought that some of the recently discovered metal-rich DA white dwarfs with dusty disks around them - also known as DAZd white dwarfs - could be formed by accretion of a minor planet. The origin of such minor planets still remains a mystery, since asteroids sufficiently close to the white dwarf would have not survived the AGB phase (Villaver \& Livio 2007). However, planet formation in these metal-rich disks is expected to be fairly efficient, thus providing a natural environment where minor planetary bodies could be formed and, ultimately, tidally disrupted to produce the observed abundance pattern in these white dwarfs (García-Berro et al. 2007). Nuclear reactions are also important in the case in which a regular $0.6 M_{\odot}$ carbon-oxygen white dwarf and a massive oxygen-neon white dwarf of $1.2 M_{\odot}$ are involved. In this case the peak temperature achieved during the coalescence is relatively high $T_{\text {peak }} \simeq 1.0 \times 10^{10}-$ see Table $1-$ enough to power carbon burning. Consequently, the chemical abundances of the Keplerian disk and of the hot corona are largely enhanced in oxygen and neon, which are the main products of carbon burning. We must add, however, a cautionary remark regarding the chemical compositions of the mergers studied here. White dwarfs are characterized in $\sim 80 \%$ of the cases by a thin hydrogen atmosphere of $\sim 10^{-4} M_{\odot}$ on top of a helium buffer of $\sim 10^{-2} M_{\odot}$. In the remaining $\sim 20 \%$ of the cases, the hydrogen atmosphere is absent. Small amounts of helium could indeed change the nucleosynthetic pattern of the hot corona in all these cases. Studying this possibility is beyond the scope of this paper and, thus, the changes in the abundances associated to burning of the helium buffer and of the atmospheric hydrogen remain to be explored.

In Fig. 3 we explore the final characteristics of the merged configuration. We start discussing the left panels of Fig. 3, which show the rotational velocity of the merger as a function of the distance to the center of the merged object. Clearly in all the cases there is a central region that rotates as rigid solid - see last column of Table 1 . This behavior has already been found in Guerrero et al. (2004) and Yoon et al. (2007) and is a consequence of the conservation of angular momentum. A differentially rotating layer is present on top of this region. This rapidly rotating region is formed by material coming from the disrupted secondary, which has been accumulated on top of the primary and thus carries the original angular moment of the secondary. Finally, a rotationally-supported disk is found for a large enough radius. The exact location where the disk begins can be easily found by looking at the left panels of Fig. 3, where the Keplerian velocity is also shown. The change in the slope of the profile of the rotational velocity clearly marks the outer edge of the compact inner object and the beginning of the disk. All the disks extend up to some solar radii - see column eight in Table 1. The stratification of surface densities of these disks can be seen in the left panels of Fig. 3, where we have plotted the surface density as a function of the distance. For the sake of comparison, the theoretical surface density of a thin disk analytical model (Livio et al. 2005 ) is also shown. Within this model the surface density of the disk should be of the form $\Sigma \propto R^{-\beta}$. We used $\beta=7 / 4$ to produce the dashed lines in the right panels of Fig. 3, very close to the value adopted by Livio et al. (2005), $\beta=3 / 2$. As can be seen in this figure for the first two fiducial mergers studied here there is a region where the analytical model and the numerical results are in good agreement. However, at long enough distances, the SPH density profile falls off more rapidly than that of the theoretical model. The agreement is poor in the case of the merger of two equal-mass $0.6 M_{\odot}$ white dwarfs. In this case, the symmetry of the system avoids the formation of a clear disk structure, instead giving rise to a rotating ellipsoid around the central compact object. Moreover, it can be shown that the angular momentum of the disk can be expressed in terms of the disk radius $R_{\text {disk }}$ 

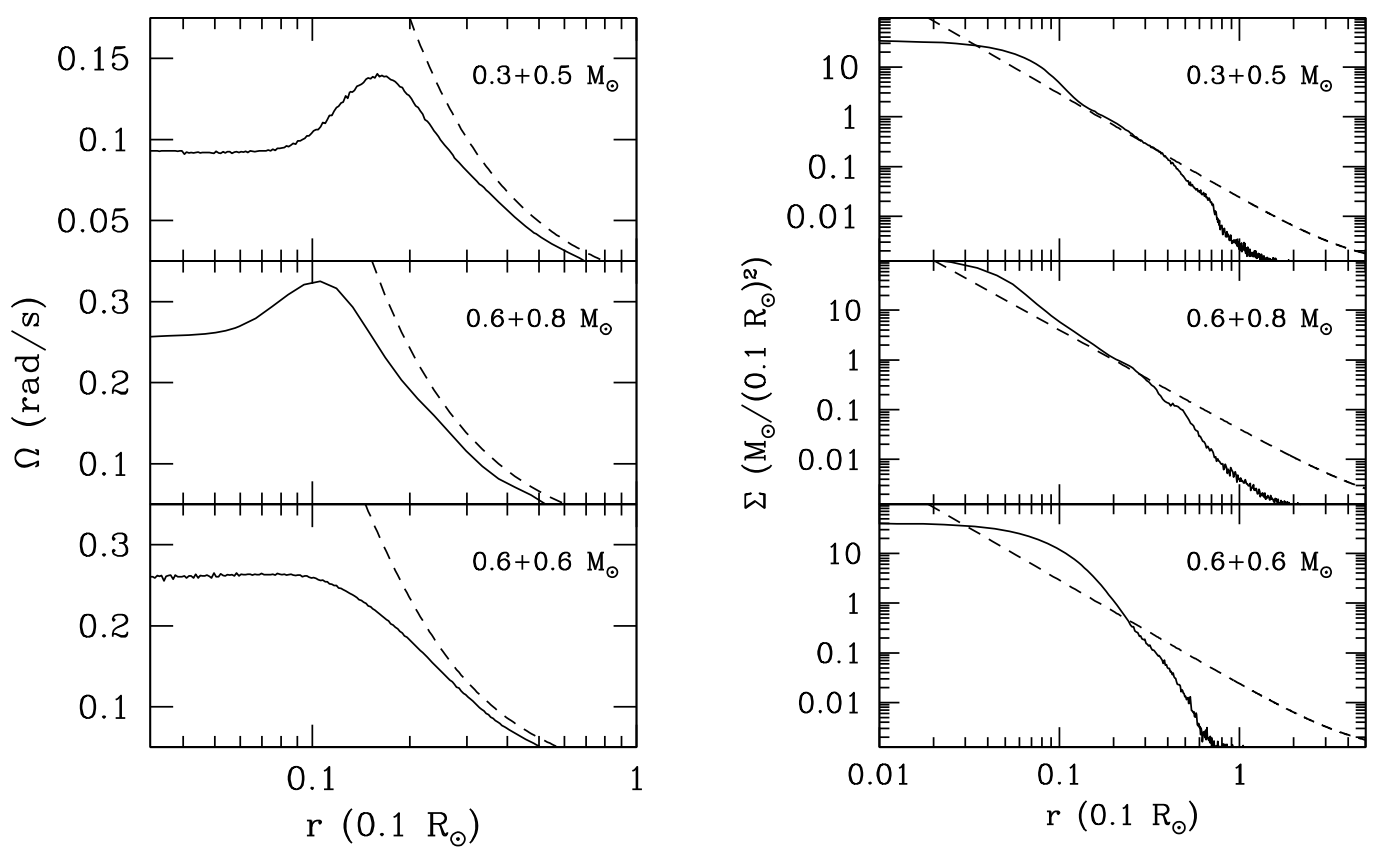

Fig. 3. Left panels: rotational velocity of the merger products as a function of the radius. For the sake of comparison the Keplerian velocity is also shown as a dashed line. Right panels: surface density profiles compared with the theoretical thin disk model profiles (dashed lines).

and the disk mass $M_{\text {disk }}$ as $J_{z}=\xi M_{\text {disk }}\left(G M_{\mathrm{WD}} R_{\text {disk }}\right)^{1 / 2}$, where $\xi=(2-\beta) /(5 / 2-\beta)=1 / 3$. The theoretical angular moments obtained using this equation agree very well with the results of our SPH simulations.

In Fig. 4 we show the temperature profiles at the end of the simulations for some of the mergers studied here. We averaged the temperatures of those particles close to the orbital plane. The average was done using cylindrical shells, and the size of these shells was chosen in such a way that each of them contains a significative number of particles. As can be seen, for the $0.3+0.5 M_{\odot}$ and the $0.4+0.8 M_{\odot}$ systems, the region of maximum temperatures occurs off-center, at the edge of the original primary, in the region of accreted and shocked material, whereas for the merger in which two equal-mass $0.6 M_{\odot}$ white dwarfs coalesce the maximum temperature occurs at the center of the merged object, as should be expected. These maximum temperatures are listed in the seventh column of Table 1. In fact, the temperature profiles shown in this figure clearly show that the cores of the primaries in the first two simulations almost remain intact and, hence, are rather cold. These cores, in turn, are surrounded by a hot envelope that corresponds to the shocked material coming from the disrupted secondary. Nuclear reactions are responsible for the observed heating of the accreted matter, initially triggered in the shocked regions.

The case in which two $0.6 M_{\odot}$ white dwarfs coalesce is somewhat different. In this case there is no hot envelope around a central object - although a local maximum of temperatures can indeed be appreciated at the edge of the rapidly spinning central object, as shown in the bottom panel of Fig. 4 - and, instead, the central region of the compact object is formed by the cores of the merging white dwarfs. Most of the temperature increase in this case stems from viscous heating since nuclear reactions are negligible because the increase in temperature of the shocked material is not enough to ignite carbon. In all these cases, a sizeable dispersion of temperatures in the outermost regions is apparent. This dispersion is due in part to this region containing some of the particles that were ejected during the first and most violent phases of the merging process.
In summary, we find that the qualitative behavior of all the mergers studied here is similar. In particular the less massive component of the binary system is disrupted in a few orbital periods. Additionally, we find that nuclear reactions are only important in the cases in which the secondary is a He white dwarf or in the case in which the primary (the accretor) is an ONe white dwarf. The total ejected mass is very small in all these cases. Finally, the overall final configuration is very similar in all them but when two equal-mass white dwarfs coalesce.

\section{Comparison with previous works}

As previously discussed, all the mergers studied here coalesce on a dynamical time scale, regardless of its mass ratio. In agreement with previous calculations, the central regions of the remnant rotate as a rigid body. On top of this rapidly spinning core, a hot corona forms and, on top of that, a heavy Keplerian disk. The question is now how the main characteristics of the merged configuration compare with those obtained in previous works? Yoon et al. (2007) computed the coalescence of a single $0.6+0.9 M_{\odot}$ binary system and the comparison is not straightforward, but our $0.6+0.8 M_{\odot}$ run is similar. The first thing to be noted is that the duration of the merger is very similar in both cases. We obtained a duration of $164 \mathrm{~s}$ and Yoon et al. (2007) obtain $\sim 150 \mathrm{~s}$. The central density of the rapidly spinning core is in both cases $\rho \sim 10^{7} \mathrm{~g} / \mathrm{cm}^{3}$. The temperature of the core is $\log T=7.9$, whereas Yoon et al. (2007) obtain $\log T \sim 4.5$, but this is due to our choice of the initial temperature of the coalescing white dwarfs, for which we adopted $T=10^{7} \mathrm{~K}$. The temperatures of the hot coronae are remarkably similar in both cases, $\log T \sim 8.5$ and 8.6, respectively. The peak temperatures attained during the merger are also very similar $-1.7 \times 10^{9} \mathrm{~K}$ and $1.6 \times 10^{9} \mathrm{~K}$, respectively. However, the temperature of the hot coronae is considerably lower in their case $T_{\max } \simeq 5.8 \times 10^{8} \mathrm{~K}$. This value has to be compared with that shown in Table $1, T_{\max } \simeq 8.7 \times 10^{8} \mathrm{~K}$. However, it should be taken into account that Yoon et al. (2007) followed the evolution of the merger for much longer times. The sizes of the resulting disk are also very similar. Yoon et al. (2007) 


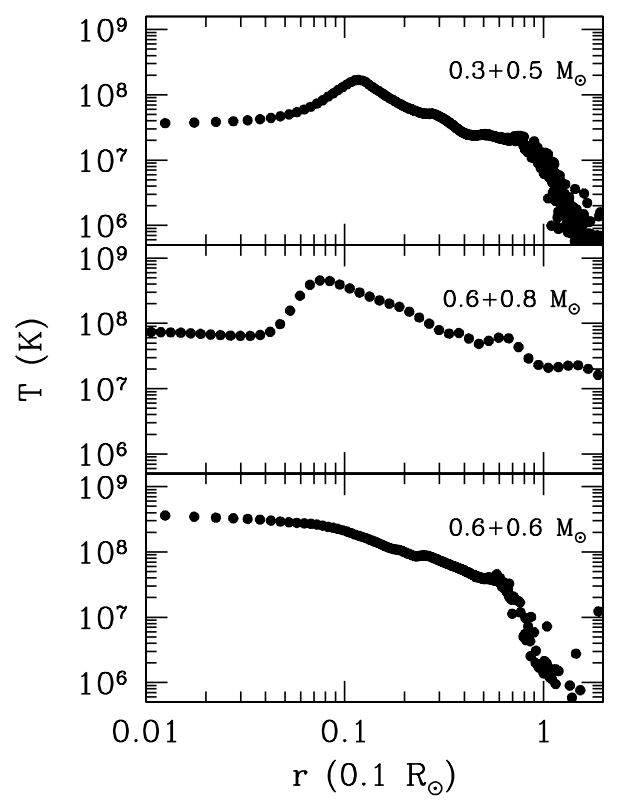

Fig. 4. Radially averaged temperature profiles as a function of radius.

obtained $8 \times 10^{9} \mathrm{~cm}$, whereas we obtain $1 \times 10^{10} \mathrm{~cm}$. Moreover, despite of the very different approaches for the artificial viscosities adopted in the work of Yoon et al. (2007) and in the present work, the rotational velocities of the central spinning object are very close, $0.21 \mathrm{~s}^{-1}$ in the case of Yoon et al. (2007), whereas we obtain $0.26 \mathrm{~s}^{-1}$. However, we find that the rotational velocities of the hot coronae are somewhat different. In particular, Yoon et al. (2007) obtained $\omega \sim 0.54 \mathrm{~s}^{-1}$, while we obtain $0.33 \mathrm{~s}^{-1}$. This could stem from the different treatment of the artificial viscosity and to the different masses of the coalescing white dwarfs.

The comparison with the results of Guerrero et al. (2004) also yields interesting results. For instance, the angular velocity of the central compact object of the $0.6+0.8 M_{\odot}$ merger in the calculations of Guerrero et al. (2004) is $0.33 \mathrm{~s}^{-1}$, somewhat larger than that obtained here. Consequently, the central density obtained in the simulations of Guerrero et al. (2004) is lower $\left(\rho \sim 6.3 \times 10^{6} \mathrm{~g} / \mathrm{cm}^{3}\right)$, because of our improved treatment of the artificial viscosity, which considerably reduces the excess of shear and, thus, translates into weaker centrifugal forces. Also, the location of the hot corona is different in the case of the simulations presented here. Specifically, the hot corona is located at $0.008 R_{\odot}$ in the simulations discussed here, whereas in the case of Guerrero et al. (2004) it was located at $0.01 R_{\odot}$. Also, the temperature of the central object is smaller in our case $\left(T \sim 7.6 \times 10^{7} \mathrm{~K}\right)$ than in the case of Guerrero et al. (2004) for which a central temperature of $T \sim 3.8 \times 10^{7} \mathrm{~K}$ was obtained, even though the initial temperatures of the coalescing white dwarfs were the same in both cases $\left(10^{7} \mathrm{~K}\right)$. Hence, our improved treatment of the artificial viscosity results in a smaller overheating and in smaller shear.

\section{Discussion}

\subsection{Comparison with theory}

To obtain a better understanding of the coalescence process and to compare our results with those theoretically expected, we numerically solved the equations of the evolution of the binary system during the mass transfer phase. The evolution of a binary system during this phase is determined by three basic physical processes, namely, gravitational wave emission, tidal torques and mass transfer. There is a wealth of literature dealing with this problem. We adopt as our starting point the analysis of Marsh et al. (2004) and the more recent formulation of Gokhale et al. (2007). Within this approach the evolution of the orbital separation $a$ is given by

$\frac{\dot{a}}{2 a}=\frac{\dot{J}_{\mathrm{GW}}}{J_{\mathrm{orb}}}-\frac{I_{1}\left(\Omega-\omega_{1}\right)}{J_{\mathrm{orb}} \tau_{1}}-\frac{I_{2}\left(\Omega-\omega_{2}\right)}{J_{\mathrm{orb}} \tau_{2}}-\left(q_{\mathrm{a}}-q\right) \frac{\dot{M}_{2}}{M_{2}}$

where $M_{1}$ and $M_{2}$ are, respectively, the masses of the accretor and of the donor, $q \equiv M_{2} / M_{1}$ is the mass ratio, $\Omega$ is the orbital velocity, $\omega_{i}$ are the spin velocities, $I_{i}$ stands for the moments of inertia, $\tau_{i}$ are the synchronization timescales, and $q_{\mathrm{a}}$ is defined as

$q_{\mathrm{a}} \equiv 1+M_{2} \frac{j_{2}-j_{1}}{J_{\text {orb }}}$

with $j_{1}$ the specific angular momentum of the matter arriving to the accretor and $j_{2}$ the angular momentum of the matter leaving the donor. In the calculations presented here, we have adopted for $j_{1}$ the expression for disk fed accretion:

$j_{1}=\sqrt{G M_{1} R_{1}}$,

whereas for $j_{2}$ we have

$j_{2} \simeq R_{2}^{2} \omega_{2}$.

The first term in Eq. (4) corresponds to the change in the orbital separation due to gravitational losses. Because of the short duration of the coalescing process, its contribution can be neglected. The second and third term describe the tidal couplings. Finally, the last term in Eq. (4) corresponds to the advected angular momentum.

The evolution of the Roche lobe radius $R_{\mathrm{L}}$ is given by

$$
\begin{aligned}
\frac{\dot{R}_{\mathrm{L}}}{2 R_{\mathrm{L}}}= & \frac{\dot{J}_{\mathrm{GW}}}{J_{\mathrm{orb}}}-\frac{I_{1}\left(\Omega-\omega_{1}\right)}{J_{\mathrm{orb}} \tau_{1}}-\frac{I_{2}\left(\Omega-\omega_{2}\right)}{J_{\mathrm{orb}} \tau_{2}} \\
& -\left(q_{\mathrm{a}}-\frac{\zeta_{R_{\mathrm{L}}}}{2}-q\right) \frac{\dot{M}_{2}}{M_{2}}
\end{aligned}
$$

where $\zeta_{\mathrm{R}_{\mathrm{L}}} \approx 0.30+0.16 q$, which is valid for $0.01 \leq q \leq 1$ (Gokhale et al. 2007). As can be seen from this set of equations, it s possible for a system to change the type of mass tranfer from stable to unstable or vice versa because $q$ has a dynamical value. Thus, to determine the exact type of mass transfer of the system it is necessary to study how $q$ evolves with time.

An expression for the mass-transfer rate is needed to solve the previous equations. The mass-transfer rate is essentially determined by the Roche lobe overfill factor, which is defined as $\Delta \equiv R_{2}-R_{\mathrm{L}}$. For the Roche lobe radius we adopt the expression of Eggleton (1983). In the case of a $0.6 M_{\odot}$ donor white dwarf a polytropic equation of state with $n=3 / 2$ can be adopted and, thus, we have (Paczyński \& Sienkiewicz 1972):

$\dot{M}_{2}=-\alpha W(\mu)\left(\frac{\Delta}{R_{2}}\right)^{3}$

with

$W(\mu)=\frac{\sqrt{\mu} \sqrt{1-\mu}}{(\sqrt{\mu}+\sqrt{1-\mu})^{4}}\left(\frac{\mu}{R_{1}}\right)^{3}$

where $\mu=M_{2} /\left(M_{1}+M_{2}\right)$ and $\alpha$ is a smoothly varying function of the stellar parameters which, following the approach of 
Gokhale et al. (2007), we have taken as a constant, freely adjustable parameter. The synchronization timescales have been computed using the expressions of Campbell (1984)

$$
\begin{aligned}
& \tau_{1}=\tau_{1}^{0}\left(\frac{M_{1}}{M_{2}}\right)^{2}\left(\frac{a}{R_{1}}\right)^{6} \\
& \tau_{2}=\tau_{2}^{0}\left(\frac{M_{2}}{M_{1}}\right)^{2}\left(\frac{a}{R_{2}}\right)^{6}
\end{aligned}
$$

where, again, the normalization factors $\tau_{1}^{0}$ and $\tau_{2}^{0}$ are freely adjustable parameter (Marsh et al. 2004; Gokhale et al. 2007). With all these inputs the equations for the evolution of the binary system - Eqs. (4) and (8) - together with the equations for the evolution of the spin angular velocities of each of the components

$\dot{\omega}_{i}=\left(\frac{j_{i}}{I_{i}}\right) \dot{M}_{i}-\left(\frac{\dot{I}_{i}}{I_{i}}\right) \omega_{i}+\frac{\Omega-\omega_{i}}{\tau_{i}}$

can be integrated. In doing so it has to be taken into account the logical limitations of the theoretical approach. In particular, the SPH results show that mass transfer is not perfectly conservative, although this assumption is fairly good - see Table 1. Moreover, stars are not point-like masses and, more important, we did not adopt an equilibrium mass-radius relationship in our analysis. These assumptions may produce marked differences between the SPH and the theoretical results. Perhaps the most critical assumption in determining the evolution of the system is assuming that an equilibrium mass-radius relation holds for both members of the binary system. In fact, at the beginning of the masstransfer episode, stars are not in equilibrium. Consequently, we adopted a different approach. In particular, when integrating Eqs. (12) we have computed for each time step the actual moment of inertia of each star. In particular, in the case of the primary white dwarf for each computed model, we looked for location of the region with maximum temperature (see Fig. 2). We then computed the mass interior to this shell and the corresponding moment of inertia. For the donor white dwarf we looked for the region that still had an approximate spherical symmetry (see Fig. 1) and we followed the same procedure as adopted for the accretor.

In Fig. 5 we compare the theoretical results - shown as a dashed line - and the SPH results - shown as dots - for the time evolution of the orbital separation and of the spin angular moment of the accretor $\left(J_{1}\right)$ and donor stars $\left(J_{2}\right)$. The three adjustable parameters adopted in the theoretical calculations are, respectively, $\alpha=145 M_{\odot} R_{\odot}^{3} / \mathrm{yr}, \tau_{1}^{0}=3.50 \times 10^{4} \mathrm{yr}$ and $\tau_{2}^{0}=4.7510^{-8}$ yr. As can be seen, the agreement is excellent during the first phases of the merger. However, we can only compare the SPH results with the theoretical expectations, while the secondary still partially preserves its initial shape. This is why we only show a reduced time interval in Fig. 5, corresponding to the first five panels in Figs. 1 and 2. For times longer than $\sim 70 \mathrm{~s}$, the secondary rapidly dissolves, hence, the approach followed here is no longer valid. It is worth realizing that $\tau_{1}^{0} \gg \tau_{2}^{0}$. This means that the synchronization timescale of the primary is much larger than that of the secondary. Accordingly, during this phase of the mass-transfer episode, the donor rapidly synchronizes, whereas the primary does not. Consequently, orbital angular moment is transferred from the orbit to the donor on a short timescale, thus reducing the orbital separation. This, in turn, increases the masstransfer rate and the final result is that the secondary is rapidly disrupted. Since the total angular momentum is conserved, the material transferred to the primary must rotate rapidly, thus producing the characteristic rotational profiles shown in the left

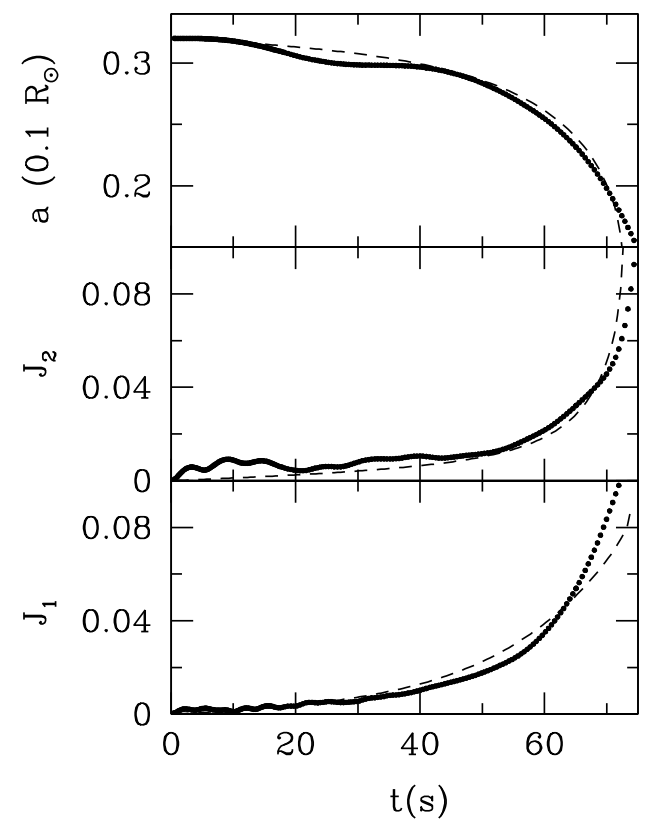

Fig. 5. A comparison of the SPH values for the orbital distance $a$ and for the spin angular moments of the donor and the accretor stars for the $0.6+0.8 M_{\odot}$ case and those obtained using a simplified model. The spin angular moments are expressed in units of $10^{51} \mathrm{~g} \mathrm{~cm}^{2} / \mathrm{s}$.

panels of Fig. 3. In summary, the results of the hydrodynamic calculations can be accurately reproduced by a simple model once all the weaknesses of the theoretical approach are correctly taken into account.

\subsection{Gravitational wave radiation}

Gravitational wave radiation from Galactic close white dwarf binary systems is expected to be the dominant contribution to the background noise in the low frequency region, which ranges from $\sim 10^{-3}$ up to $\sim 10^{-2} \mathrm{~Hz}$ (Bender et al. 1998). Moreover, since a sizeable amount of mass is transferred from the donor star to the primary at considerable speeds during the merging process, the gravitational wave signal is expected to be detectable by LISA (Guerrero et al. 2004; Lorén-Aguilar et al. 2005). It is thus important to characterize which would be the gravitational wave emission of the white dwarf mergers studied here and to assess the feasibility of dectecting them.

To compute the gravitational wave pattern, we proceed as in Lorén-Aguilar et al. (2005). In particular, we use the weak-field quadrupole approximation (Misner et al. 1973):

$h_{j k}^{\mathrm{TT}}(t, \boldsymbol{x})=\frac{2 G}{c^{4} d} \frac{\partial^{2} Q_{j k}^{\mathrm{TT}}(t-R)}{\partial t^{2}}$

where $t-R=t-d / c$ is the retarded time, $d$ the distance to the observer, and $Q_{j k}^{\mathrm{TT}}(t-R)$ the quadrupole moment of the mass distribution, which is given by

$\ddot{Q}_{j k}^{\mathrm{TT}}(t-R)=\int \rho(\boldsymbol{x}, t-R)\left(x^{j} x^{k}-\frac{1}{3} x^{2} \delta_{j k}\right) \mathrm{d}^{3} x$.

To calculate the quadrupole moment of the mass distribution using SPH particles, Eq. (14) must be discretized according to the 


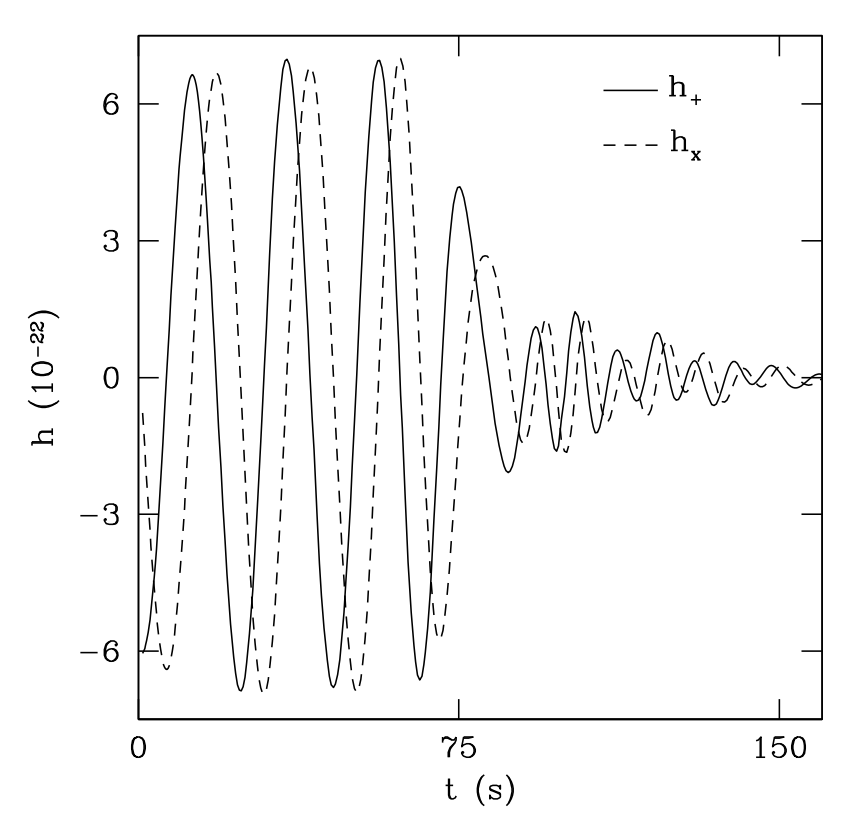

Fig. 6. Gravitational wave emission from the merger of a $0.6+0.8 M_{\odot}$ close white dwarf binary system. The dimensionless strains $h_{+}$and $h_{\times}$ are measured in units of $10^{-22}$. The source has been assumed to be at a distance of $10 \mathrm{kpc}$.

following expression

$$
\begin{aligned}
\ddot{Q}_{j k}^{\mathrm{TT}}(t-R) & \approx P_{i j k l}(\boldsymbol{N}) \sum_{p=1}^{n} m(p)\left[2 \boldsymbol{v}^{k}(p) \boldsymbol{v}^{l}(p)\right. \\
& \left.+\boldsymbol{x}^{k}(p) \boldsymbol{a}^{l}(p)+\boldsymbol{x}^{l}(p) \boldsymbol{a}^{k}(p)\right]
\end{aligned}
$$

where

$$
\begin{aligned}
P_{i j k l}(N) \equiv & \left(\delta_{i j}-N_{i} N_{k}\right)\left(\delta_{j l}-N_{j} N_{l}\right) \\
& -\frac{1}{2}\left(\delta_{i j}-N_{i} N_{j}\right)\left(\delta_{k l}-N_{k} N_{l}\right)
\end{aligned}
$$

is the transverse-traceless projection operator onto the plane orthogonal to the outgoing wave direction, $N, m(p)$ is the mass of each SPH particle, and $\boldsymbol{x}(p), \boldsymbol{v}(p)$, and $\boldsymbol{a}(p)$ are, respectively, its position, velocity, and acceleration.

Using this prescription, the corresponding strains for the $0.6+0.8 M_{\odot}$, which is a representative case, are shown in Fig. 6 . As can be seen, the gravitational waveforms rapidly vanish in a couple of orbital periods and the gravitational wave emission during the coalescence phase does not have a noticeably strong peak. Hence, the gravitational wave emission is dominated by the chirping phase, in agreement with the findings of Lorén-Aguilar et al. (2005). Moreover, the gravitational waveforms obtained here are very similar to those computed by Lorén-Aguilar et al. (2005) and, thus, do not depend appreciably on the number of particles used to calculate them. This is because most of the emission of gravitational waves comes from the regions in which the SPH particles change appreciably their velocities, and these regions were well resolved in both sets of simulations. Higher order terms of gravitational wave emission could be included in calculating the strains. These terms include the current-quadrupole and the mass octupole. It has been shown (Schutz \& Ricci 2001) that, for the first of these to be relevant, an oscillating angular momentum distribution with a dipole moment along the angular momentum axis is needed. Consequently,

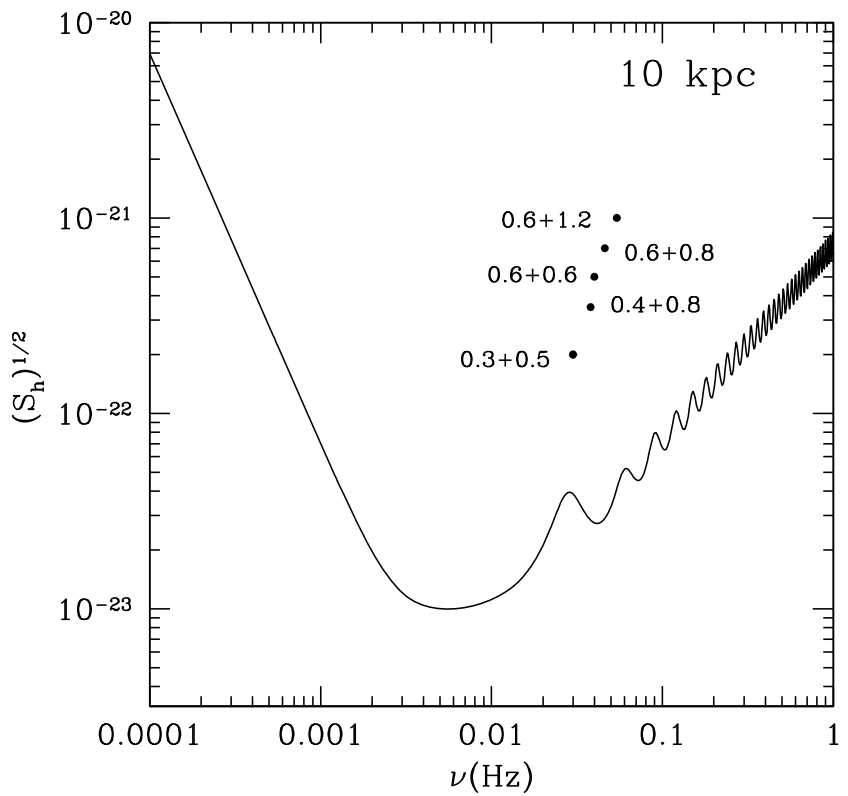

Fig. 7. A comparison of the signal produced by the close white dwarf binary systems studied here when a distance of $10 \mathrm{kpc}$ is adopted, with the spectral distribution of noise of LISA. The spectral distribution of noise of LISA is for a one-year integration period. We have adopted a signal-to-noise ratio $\eta=5$.

in our calculations only the mass octupole should be considered in the best of the cases. Within this approximation, a term close to $v / c \sim 10^{-3}$ would be added to the derived strains. We performed a post-processing of our simulations and we find that the octupole emission is $h \sim 10^{-24}$, just above the numerical noise $\left(h \sim 10^{-25}\right)$, but in either case totally negligible. Since the gravitational wave signal is dominated by that of the inspiralling phase, to assess the feasibility of detecting it using gravitational wave detectors, we assumed that the orbital separation of the binary system is that of the last stable orbit. Furthermore, we also assumed that the integration time of LISA will be one year. It is then straightforward to demonstrate that, during this time interval, the variation in the orbital separation is negligible. With these assumptions the double white-dwarf binary system basically radiates a monochromatic wave, and it is easy to assess the feasibility of detecting the signal produced by the coalescence of close binary white dwarf systems. This is done in Fig. 7 where we show the strength of the resulting signals and compare them with the spectral distribution of noise of LISA, when a distance of $10 \mathrm{kpc}$ is adopted. As can be seen in this figure, all the systems are well inside the detectability region, hence, LISA should be able to distinguish them from Galactic. However, the typical rate of white dwarf mergers is rather small, $\sim 9 \times 10^{-3} \mathrm{yr}^{-1}$ (Nelemans 2003); consequently, although there is an uncertainty of a factor of 5 in the rate of white dwarf mergers, the expected detection rate is small as well.

\subsection{Fallback luminosities}

Another potential observational signature of the mergers studied here is the emission of high-energy photons from the fallback material in the aftermath of the coalescence itself. We have already shown that, as a result of the merger of two white dwarfs of different masses, most of the SPH particles of the disrupted secondary form a Keplerian disk. These SPH particles have 


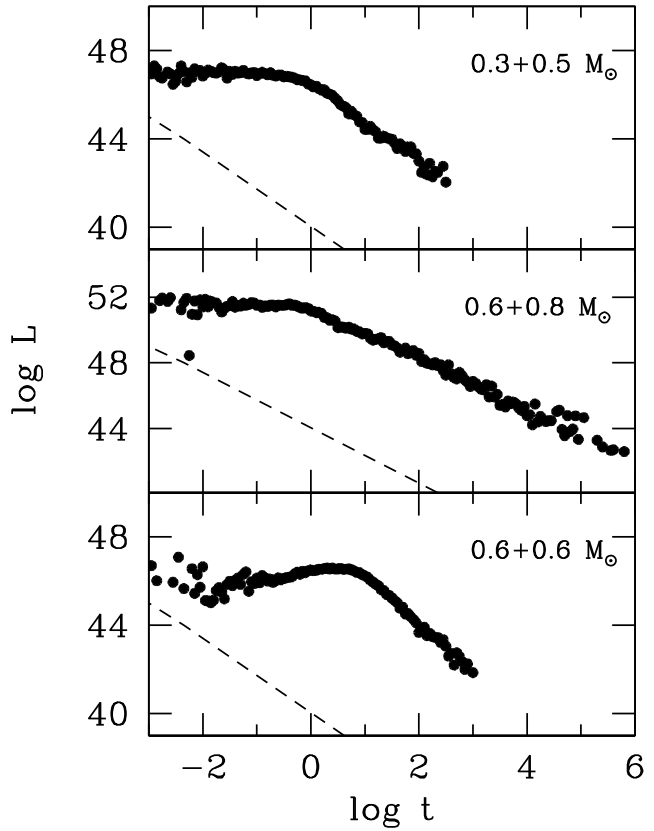

Fig. 8. Fallback accretion luminosity for our three fiducial double white dwarf mergers. The units of time are seconds, whereas those of luminosities are erg/s. A straight line with slope 5/3 is shown for the sake of comparison.

circularized orbits. However, as it occurs in the coalescence of double neutron stars, some material of the secondary is also found to be in highly eccentric orbits. After some time, this material will most likely interact with the recently formed disk. As discussed in Rosswog (2007), the timescale for this is not set by viscous dissipation but, instead, by the distribution of eccentrities. We followed the model proposed by Rosswog (2007) closely and calculated the accretion luminosity obtained from the interaction of the stellar material with high eccentricities with the newly formed disk by assuming that the kinetic energy of these particles is dissipated within the radius of the debris disk.

In Fig. 8 we have plotted the accretion luminosities as a function of time for our three fiducial cases. We emphasize that these luminosities were computed assuming that the highly eccentric particles lose all their kinetic energy when interacting with the disk, for which we adopt the radius obtained by the end of our SPH simulations, which are those shown in Table 1. Moreover, only a fraction of this energy will be released in the form of highenergy photons. Thus, the results shown in Fig. 8 can be regarded as an upper limit for the actual luminosity of high-energy photons. Although the luminosities are lower than those typically obtained for the merger of double neutron stars - which are typically $\sim 10^{52} \mathrm{erg} / \mathrm{s}$ - white-dwarf mergers predict a very similar time dependence $\left(\propto t^{5 / 3}\right)$.

This is an important result because it shows that observations of high-energy photons can help in detecting the gravitational wave signal radiated by these systems. In fact, the detection of the gravitational waves arising from the merger of white dwarfs is a tough task because, as previously explained, the signal is dominated by the inspiralling phase and the waveforms do not have a prominent peak before the ringdown phase. Thus a combined strategy in which optical, UV, X-ray, and gravitational wave detectors are used could be very useful.

\subsection{Long-term evolution}

We have already shown that non-explosive nuclear burning takes place during the merging phase. However, this does not necessarily mean that such an explosion could not take place due to mass accretion from the disk at late times. If mass acccretion occurs at rates less than $10^{-6} M_{\odot} \mathrm{yr}^{-1}$, then central carbon ignition is possible and a type Ia supernova (SNIa) is the most probable outcome. On the other hand, if the accretion rates are higher than this value, then off-center carbon ignition is the most probable outcome, giving rise to an inward propagating burning flame and an ONe white dwarf is likely to be formed (Nomoto \& Iben 1986; García-Berro \& Iben 1994; Ritossa et al. 1999) which might eventually form a neutron star by accretion-induced collapse (Saio \& Nomoto 1985; Woosley \& Weaver 1986; Gutiérrez et al. 1996, 2005). However, once the disk has been formed, angular momentum viscous transfer is relevant and the hydrodynamical timescale of the disk becomes very large. Consequently, the subsequent evolution of the disk cannot be followed using an SPH code. However, some estimates of the accretion rate can still be done by considering the typical viscous transport timescales.

The typical viscous transport timescale is (Mochkovitch \& Livio 1989, 1990)

$\tau_{\text {visc }}=\left(\frac{1}{T} \frac{\mathrm{d} T}{\mathrm{~d} t}\right)^{-1}$

where $T$ is the rotational kinetic energy and

$$
\frac{\mathrm{d} T}{\mathrm{~d} t}=-\int\left(\frac{\partial \Omega}{\partial r}\right)^{2} r^{2} \eta(r) \mathrm{d}^{3} r
$$

is its rate of change. In this expression $\Omega$ is the angular velocity, $r$ the radial cylindrical coordinate, and $\eta$ the (physical) viscosity parameter, that depends on the viscous mechanism. If the disk is laminar and the viscosity is that of degenerate electrons, then $\eta=2.0 \times 10^{-5} \rho^{5 / 3} \mathrm{~g} / \mathrm{cm} \mathrm{s}$ (Durisen 1973; Itoh et al. 1987) and the associated accretion rates can be obtained taking into account that

$$
\dot{T} \sim \frac{G M_{*} \dot{M}}{R_{*}}
$$

where $M_{*}$ and $R_{*}$ are the mass and radius of the central object. If, instead, the disk is turbulent the classical approximation of Shakura \& Sunyaev (1973) is valid. Within this approximation the viscous timescale is given by

$\tau_{\text {visc }}=\alpha^{-1}\left(\frac{R_{\text {disk }}}{H}\right)\left(\frac{R_{\text {disk }}}{c_{\mathrm{s}}}\right)$

where $\alpha \sim 0.1$ is the standard viscosity, $c_{\mathrm{s}}$ the sound speed, $R_{\mathrm{disk}}$ the radius of the disk and $H$ the disk half thickness. Both the radius of the disk and its half thickness are listed in Table 1 for each one of the simulations presented here. The accretion rate is then given by

$\dot{M} \simeq \frac{M_{\text {disk }}}{\tau_{\text {visc }}}$

In order to check that the disk is turbulent we have computed the Richardson number

$\mathrm{Ri}=\frac{\left(\frac{g^{\text {eff }}}{c_{\mathrm{s}}}\right)\left(1-\frac{\gamma}{\Gamma}\right)}{\left(r \frac{\mathrm{d} \Omega}{\mathrm{d} r}\right)}$. 


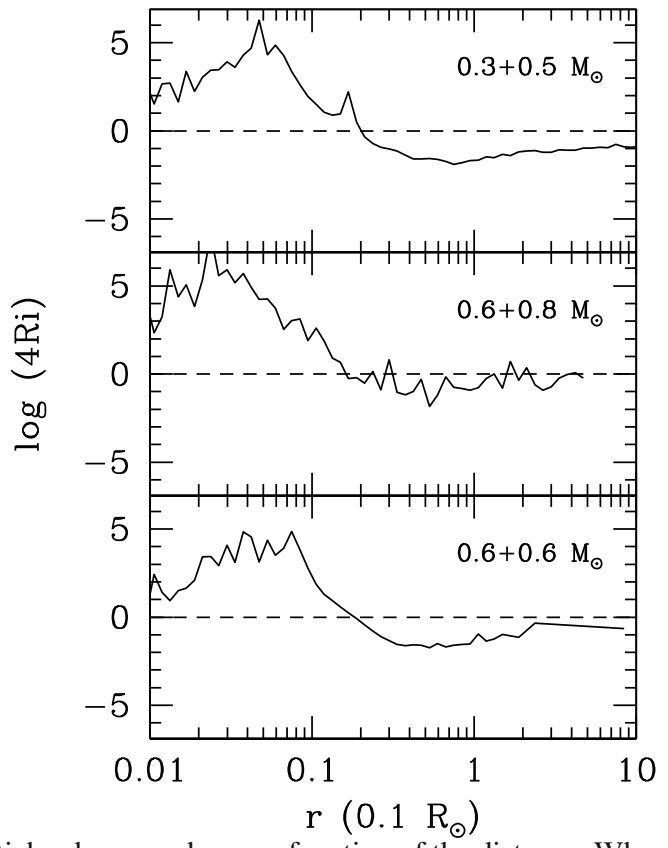

Fig. 9. Richardson number as a function of the distance. When $\mathrm{Ri}>1 / 4$, the disk is turbulent. The horizontal dashed line corresponds to $\mathrm{Ri}=1 / 4$.

In this expression $g^{\text {eff }}$ is the effective gravity, that is, the real gravity minus the centrifugal force; $\gamma$ is the logarithmic derivative of the pressure with respect to the density; $\Gamma$ is the adiabatic index; and the rest of the symbols have their usual meaning. We have chosen $\gamma=1.4$ and $\Gamma=5 / 3$. If the Richardson number is smaller than $1 / 4$, stability against turbulence is guaranteed. If this is not the case, the disk may be turbulent, since this is only a necessary condition, but $\mathrm{Ri}>1 / 4$ is a good indication that turbulence occurs. In Fig. 9 we show the Richardson number as a function of the radial coordinate for the three fiducial cases described here. As can be seen in this figure, the condition $\mathrm{Ri}>1 / 4$ is satisfied in the innermost regions of the disk, up to distances $\sim 0.2 R_{\odot}$. Thus, the innermost regions of the disk are potentially turbulent and it is likely that the accretion rate is the one given by Eq. (21). Nevertheless, Eq. (17) provides a safe lower limit for the typical transport timescale of the disk. The total rotational kinetic luminosity dissipated, $\dot{T}$, and the corresponding timescales using this approach are given in the left section of Table 3. For the case in which the laminar viscosity is used the resulting accretion rates turn out to be $\lesssim 10^{-12} M_{\odot} / \mathrm{yr}$, and consequently central carbon ignition leading to a SNIa is possible. When the classical Shakura \& Sunyaev (1973) expression is adopted the accretion rates are shown in the right section of Table 3 . As can be seen these accretion rates turn out to be very high. There are experimental and theoretical reasons to suspect that the central object will not be able to accrete material from the surrounding disk at these rates. From a theoretical perspective, it is rather clear that the accretion rates exceed the Eddington limit, which is order of $10^{-5} M_{\odot} \mathrm{yr}^{-1}$. Additionally, and from a experimental point of view, there is growing evidence ( $\mathrm{Ji}$ et al. 2006) that hydrodynamic turbulence cannot transport angular momentum effectively in astrophysical disks, even at very large Reynolds numbers, leaving the magnetorotational instability as the only possible way to lose angular momentum.

Yoon et al. (2007) have systematically explored the conditions for avoiding off-center carbon ignition in the merged configurations resulting from the coalescence of two $\mathrm{CO}$ white dwarfs. They computed the evolution of the central remnant of
Table 3. Typical viscous timescales (in years), dissipated kinetic energies (in erg/s), and accretion rates (in $M_{\odot} \mathrm{yr}^{-1}$ ) when the laminar or the turbulent viscosity are used.

\begin{tabular}{cccccc}
\hline \hline & \multicolumn{3}{c}{ Laminar } & \multicolumn{2}{c}{ Turbulent } \\
\hline Run & $\tau$ & $\dot{T}$ & $\dot{M}$ & $\tau$ & $\dot{M}$ \\
\hline $0.3+0.5$ & $3 \times 10^{11}$ & $1 \times 10^{29}$ & $2 \times 10^{-14}$ & $7.6 \times 10^{-4}$ & 43 \\
$0.4+0.8$ & $1 \times 10^{11}$ & $5 \times 10^{29}$ & $8 \times 10^{-14}$ & $1.1 \times 10^{-2}$ & 63 \\
$0.6+0.6$ & $1 \times 10^{11}$ & $6 \times 10^{29}$ & $1 \times 10^{-13}$ & $2.0 \times 10^{-4}$ & 560 \\
$0.6+0.8$ & $5 \times 10^{10}$ & $3 \times 10^{30}$ & $4 \times 10^{-13}$ & $1.2 \times 10^{-2}$ & 62 \\
$0.6+1.2$ & $3 \times 10^{8}$ & $7 \times 10^{32}$ & $8 \times 10^{-11}$ & $1.0 \times 10^{-2}$ & 75 \\
\hline
\end{tabular}

the coalescence of a $0.9+0.6 M_{\odot}$ binary white dwarf, adopting a realistic initial model, which includes the differentially rotating primary, the hot envelope we also find in our simulations and the centrifugally supported accretion disk. Our final configurations closely resemble those found by these authors and, consequently, the same conclusions obtained in this paper hold. In particular, since in our models the maximum temperature of the hot envelope is lower than the carbon ignition temperature and the mass-accretion rate from the Keplerian disk is possibly smaller than the critical one $\left(\dot{M} \simeq 5.0 \times 10^{-6} M_{\odot} \mathrm{yr}^{-1}\right)$, it is probable that at least some of the merged configurations with a total mass exceeding Chandrasekhar's mass may be considered good candidates for Type Ia supernovae progenitors.

\section{Conclusions}

We have performed several high-resolution smoothed particle hydrodynamics simulations of coalescing white dwarfs for a broad range of masses and chemical compositions of the coalescing white dwarfs, which includes $\mathrm{He}, \mathrm{CO}$, and $\mathrm{ONe}$ white dwarfs. Such a parametric study using a large number of particles ( $4 \times 10^{5} \mathrm{SPH}$ particles) had never been done before. Previous works on the subject used a considerably smaller number - by a factor of 10 - of SPH particles (Guerrero et al. 2004), or did not explore the full range of masses and chemical compositions of interest (Yoon et al. 2007) - only one merger was computed, that of a $0.9+0.6 M_{\odot}$ double white dwarf. In addition, we included a refined treatment of the artificial viscosity. In particular, we used an artificial viscosity formulation that is oriented on Riemannsolvers (Monaghan 1997), together with an additional switch to suppress the excess of viscosity (Balsara 1995). With this treatment the dissipative terms are only applied in those regions of the fluid in which they are really needed to resolve a shock. This refined treatment of the artificial viscosity overcomes some of the problems found in our previous simulations (Guerrero et al. 2004).

In all cases, the merged configuration consists of a compact central object surrounded by a hot corona with spheroidal shape and a self-gravitating Keplerian disk around it. For those cases in which two white dwarfs of different masses are involved, the resulting disk can be considered as a thin disk, whereas for the $0.6+0.6 M_{\odot}$ case, we found that the resulting final configuration resembles a rotating ellipsoid around the central object with a much more modest disk. The peak temperatures attained during the merging process are lower than those found by Guerrero et al. (2004) and in line with that found by Yoon et al. (2007) for the case of a $0.9+0.6 M_{\odot}$ merger. We also confirm the results obtained in previous works (Guerrero et al. 2004: Lorén-Aguilar et al. 2005; Yoon et al. 2007) and find that, only when one of the merging white dwarfs is a He white dwarf, nuclear reactions are relevant. However, none of the cases studied here show any 
explosive behavior during the merging phase. Furthermore, no essential differences are found when the chemical abundances obtained here using an enhanced spatial resolution and a refined prescription for the artificial viscosity and those obtained in previous works (García-Berro et al. 2007) are compared. The chemical composition of the disks formed by the coalescence of a He white dwarf with $\mathrm{CO}$ white dwarfs shows an enrichment in heavy elements like $\mathrm{Ca}, \mathrm{Mg}, \mathrm{S}, \mathrm{Si}$, and $\mathrm{Fe}$ and constitute a natural environment where planets and asteroids can be formed. This could explain the anomalous abundances of metals found in several hydrogen-rich white dwarfs with dusty disks around them and that have been attributed to the impact of asteroids (Jura 2003), since it is quite unlikely that such asteroids could survive the red giant phase.

We also compared the results of our hydrodynamical calculations with the theoretical expectations and found a satisfactory agreement when the synchronization timescale of the disrupted secondary is much shorter than that of the primary. In this case the rate of change of the orbital distance and the corresponding spins of both the donor star and of the accretor are reproduced with a high degree of accuracy. We have shown as well that the emission of gravitational waves from these kind of systems is strong enough to be obervable by LISA and that the corresponding waveforms do not depend much on the resolution employed in the hydrodynamical calculations and, thus, that these waveforms are robust.

We also computed the possible emission of high-energy photons produced in the aftermath of the merger. This high-energy emission is a consequence of the interaction of the material with highly eccentric orbits, which are produced during the first and most violent phases of the merger with the resulting disk, which is formed by particles with circularized orbits, and found that the typical luminosities are $\sim 10^{49} \mathrm{erg} / \mathrm{s}$, although the precise value of the peak luminosity depends closely on the masses of the coalescing white dwarfs. The time dependence of the high-energy emission is $\propto t^{5 / 3}$, a behavior also present in the coalescence of double neutron stars and neutron stars and black holes (Rosswog 2007). The detection of this high-energy burst would eventually help in detecting the gravitational waves suposedly radiated during the merger.

With respect to the long-term evolution of the mergers, we have found that all the disks produced during the coalescence are potentially turbulent. This result implies that very high accretion rates are expected from the disk onto the primary. Despite our crude approximations, it is thus quite likely that these accretion rates would lead to an off-center carbon ignition, although an in-depth study remains to be done. However, since our final configurations closely resemble those found by Yoon et al. (2007), it is as probable that at least some of our merged configurations may be considered good Type Ia supernovae candidate progenitors. A detailed calculation of the evolution of the resulting disks, including an accurate description of the mechanisms of angular momentum transport, must therefore be done. Unfortunately this task is far beyond the current possibilities of SPH techniques.

Acknowledgements. Part of this work was supported by the MEC grants AYA2008-04211-C02-01, ESP2007-61593, and by the AGAUR.

\section{References}

Balsara, D. S. 1995, J. Comp. Phys., 121, 357

Barnes, J., \& Hut, P. 1986, Nature, 324, 446

Bender, P. L., et al. 2000, in LISA: a conerstone mission for the observation of gravitational waves, ESA-SCI(2000)11, System and Technology Study Report
Benz, W. 1990, in The numerical modelling of nonlinear stellar pulsations: problems and prospects, ed. J. R. Buchler (Dordrecht: Kluwer Academic Publishers), 269

Benz, W., Cameron, A. G. W., \& Bowers, R. L. 1989a, in White Dwarfs, Proc. of the ed. G. Wegner, LNP 328 (Berlin: Springer-Verlag), IAU Coll., 114, 511

Benz, W., Thielemann, F. K., \& Hills, J. G. 1989b, ApJ, 342, 986

Benz, W., Cameron, A. G. W., Press, W. H., \& Bowers, R. L. 1990, A\&A, 348, 647

Campbell, C. G. 1984, MNRAS, 207, 433

Clayton, G. C., Geballe, T. R., Herwig, F., Fryer, C., \& Asplund, M. 2007, ApJ, 662,1220

Clement, M. J. 1974, ApJ, 194, 709

D’Souza, M. C. R.. Motl, P. M., Tohline, J. E., \& Frank, J. 2006, ApJ, 643, 381

Durisen, R. H. 1973, ApJ, 183, 205

Eggleton, P. P. 1983, ApJ, 268, 368

García-Berro, E., \& Iben, I. 1994, ApJ, 434, 306

García-Berro, E., Lorén-Aguilar, P., Pedemonte, A. G., et al. 2007, ApJ, 661, L179

Gingold, R. A., \& Monaghan, J. J. 1977, MNRAS, 181, 375

Gokhale, V., Peng, X. M., \& Frank, J. 2007, ApJ, 655, 1010

Guerrero, J., García-Berro, E., \& Isern, J. 2004, A\&A, 257

Gutiérrez, J., García-Berro, E., Iben, I., et al. 1996, 459, 701

Gutiérrez, J., Canal, R., \& García-Berro, E. 2005, A\&A, 435, 231

Iben, I., \& Tutukov, A. V. 1984, ApJS, 54, 335

Itoh, N., Totsuji, H., Ichimaru, S., \& Dewitt, H. E. 1979, ApJ, 234, 1079

Itoh, N., Kohyama, Y., \& Takeuchi, H. 1987, ApJ, 317, 733

Itoh, N., Hayashi, H., Nishikawa, A., \& Kohyama, Y. 1996, ApJS, 102, 411

Izzard, R. G., Jeffery, C. S., \& Lattanzio, J. 2007, A\&A, 470, 661

Ji, H., Burin, M., Schartman, E., \& Goodman, J. 2006, Nature, 444, 343

King, A. R., Pringle, J. E., \& Wickramasinghe, D. T. 2001, MNRAS, 320, L45

Livio, M., Pringle, J. E., \& Wood, K. 2005, ApJ, 632, 37L

Lorén-Aguilar, P., Guerrero, J., Isern, J., Lobo, J. A., \& García-Berro, E. 2005, MNRAS, 356, 627

Lucy, L. B. 1977, AJ, 82, 1013

Marsh, T. R., Nelemans, G., \& Steeghs, D. 2004, MNRAS, 350, 113

Misner, C. W., Thorne, K. S., \& Wheeler, J. A. 1973, Gravitation, ed. W. H. Freeman (New York)

Mochkovitch, R., \& Livio, M. 1989, A\&A, 209, 111

Mochkovitch, R., \& Livio, M. 1990, A\&A, 236, 378

Monaghan, J. J. 2005, Rep. Prog. Phys., 68, 1703

Monaghan, J. J. 1997, J. Comp. Phys., 136, 298

Monaghan, J. J., \& Gingold, R. A. 1983, J. Comp. Phys., 52, 374

Monaghan, J. J., \& Lattanzio, J. C. 1985, A\&A, 149, 135

Morris, J. P., \& Monaghan, J. J. 1997, J. Comp. Phys., 136, 41

Motl, P. M., Frank, J., Tohline, J. E., \& D'Souza, M. C. R. 2007, ApJ, 670, 1314

Nelemans, G. 2003, in The Astrophysics of Gravitational Wave Sources, AIP Conf. Proc., 686, 263

Nelemans, G., Portegies Zwart, S. F., Verbunt, F., \& Yungelson, L. R. 2001a, A\&A, 368, 939

Nelemans, G., Yungelson, L. R., Portegies Zwart, S. F., \& Verbunt, F. 2001b, A\&A, 365, 491

Nomoto, K., \& Iben, I. 1985, A\&A, 297, 531

Paczyński, B., \& Sienkiewicz, R. 1972,, Acta Astron., 22, 73

Postnov, K. A., \& Yungelson, L. R. 2006, Living Rev. in Relativity, 9(6)

Price, D. J. 2007, PASA, 24, 159

Rasio, F. A., \& Shapiro, S. L. 1995, ApJ, 438, 887

Rauscher, T., \& Thielemann, F. K. 2000, Atom. and Nucl. Data Tabl., 75, 1

Ritossa, C., García-Berro, E., \& Iben, I. 1996, ApJ, 460, 489

Ritossa, C., García-Berro, E., \& Iben, I. 1999, ApJ, 515, 381

Rosswog, S. 2007, MNRAS, 376, L48

Saio, H., \& Nomoto, K. 1985, A\&A, 150, L21

Schmidt, G. D., Bergeron, P., Liebert, J., \& Saffer, R. A. 1992, ApJ, 394, 603

Segretain, L., Chabrier, G., Hernanz, M., García-Berro, E., \& Isern, J. 1994, ApJ, 434,641

Segretain, L., Chabrier, G., \& Mochkovitch, R. 1997, ApJ, 481, 355

Serna, A., Alimi, J. M., \& Chieze, J. P. 1996, ApJ, 461, 884

Shakura, N. I., \& Syunyaev, R. A. 1973, A\&A, 24, 337

Schutz, B. F., \& Ricci, F. 2001, in Gravitational waves, ed. I. Ciufolini, V. Gorini, U. Moschella, \& P. Fré, Institute of Physics (Bristol), 11

Villaver, E., \& Livio, M. 2007, ApJ, 661, 1192

Webbink, R. F. 1984, ApJ, 277, 355

Woosley, S. E., \& Weaver, T. A. 1986, ARA\&A, 24, 205

Yoon, S. C., Podsiadlowski, P., \& Rosswog, S. 2007, MNRAS, 380, 933 